\title{
Multi-scale overlapping domain decomposition to consider local effects in the analysis of pipes
}

\author{
By \\ R. Emre Erkmen ${ }^{1}$ Magdi Mohareb ${ }^{2}$ and Ashkan Afnani ${ }^{3}$ \\ ${ }^{1}$ Lecturer, School of Civil and Environmental Engineering, University of Technology, \\ Sydney, NSW 2007, Australia E-mail: emre.erkmen@uts.edu.au \\ ${ }^{2}$ Professor, Department of Civil Engineering, University of Ottawa, Ottawa, Ontario, \\ K1N6N5, Canada. E-mail: mmohareb@uottawa.ca \\ ${ }^{3}$ Graduate Research Assitant, School of Civil and Environmental Engineering, University \\ of Technology, Sydney, NSW 2007, Australia E-mail: emre.erkmen@uts.edu.au
}

*Corresponding Author: Dr R.E. Erkmen, School of Civil and Environmental Engineering, University of Technology, Sydney, NSW 2007, Australia.

Phone: +61 29514 9769; Fax: +61 29514 2633; Email: emre.erkmen@uts.edu.au

Submitted to: International Journal of Structural Stability and Dynamics, $17^{\text {th }}$ March 2015

Revised on the $30^{\text {th }}$ March 2015 


\begin{abstract}
Elevated pipelines are commonly encountered in petro-chemical and industrial applications. Within these applications, pipelines normally span hundreds of meters and are thus analysed using beam-type one-dimensional finite elements when the global behaviour of the pipeline is sought at a reasonably low computational cost. Standard beam-type elements, while computationally economic, are based on the assumption of rigid cross-section. Thus, they are unable to capture the effects of cross-sectional localized deformations. Such effects can be captured through shell-type finite element models. For long pipelines, shell models become prohibitively expensive. Within this context, the present study formulates an efficient numerical modelling which effectively combines the efficiency of beam-type solutions while retaining the accuracy of shell-type solutions. An appealing feature of the model is that it is able to split the global analysis based on simple beam-type elements from the local analysis based on shell-type elements. This is achieved through domain-decomposition procedure within the framework of the bridging multi-scale method of analysis. Solutions based on the present model are compared to those based on full shell-type analysis. The comparison demonstrates the accuracy and efficiency of the proposed method.
\end{abstract}




\section{Introduction}

Thin-walled pipes are widely used in industrial applications. Usually, they are susceptible to buckling and it is important to accurately predict their nonlinear response. Pipes usually span much larger distances in comparison to their cross-sectional dimensions. As such, beam-type elements are commonly adopted in their analysis. Standard beam-type elements, however, are based on the assumption of rigid cross-section and thus, cannot consider the deformations of the cross-section such as local buckling [1] and only allow considerations of the global behaviour such as flexural-buckling [2]. In contrast, Shelltype finite elements can capture local effects. The buckling response for long pipes under combinations of bending, axial force, and external pressure using shell analyses were investigated in [3-5]. On the other hand shell elements are computationally more expensive and time consuming, and for typical pipeline networks spanning hundreds of meters, such shell analyses become impractical.

Research on computational mechanics has been increasingly focusing on adaptive numerical analysis strategies such as mesh-free methods e.g. [6-8], Generalized-FEM [911] and Multi-scale methods [12-17], which improve the efficiency and accuracy of the numerical results by refining the model only where required and without changing the global simpler model of the whole structure. Common to these numerical methods is that the partition of unity concept is exploited to allow overlapping decompositions of the analysis domain so that a local enrichment can be seamlessly incorporated [18-21]. In various types of problems which naturally give rise to multiple scales in the deformation fields, such as crack propagation e.g., [22], or localized damage problems e.g., [23] multiscale numerical analysis techniques have been effectively used. In particular, the Bridging multi-scale method, which was originally developed to enrich the nodal values of the FEM solution with mesh-free solution [24], provides a basis to couple problems 
based on two different physical assumptions. The appealing feature of the Bridging multiscale method is that it can split the global analysis, which is based on simplified assumptions, from the local analysis which requires more sophisticated modelling. Bridging multi-scale method was previously used to incorporate nano-mechanics and atomistic behaviour into the local model e.g., [25-27], and in strain localization problems considering micro-polar continuum model with different levels of resolution e.g., [28].

In pipe buckling behaviour, the interaction of local with global modes also gives rise to multiple scales in the deformation fields. In order to capture the effect of local deformations, shell formulations have been utilised in the past e.g., [29-32]. Localized plasticity effects have also been incorporated into pipeline analysis through generalized plasticity models [33]. In order to capture ovalization in pipe elbows, efficient beam type formulations were developed in [34-36]. Recently, Erkmen [38] developed an analysis procedure based on the Bridging multi-scale method of Liu et al [25-28], in order to incorporate local deformation effects in the analysis of thin-walled members. This approach allows employment of two kinematic models within the numerical analysis, and while simple beam-type elements are used for the analysis of the overall structure, more sophisticated shell-type elements are employed for the local fine-scale analysis in a relatively narrow span of the member. In the present study, we extend the procedure developed in Erkmen [38] for the elasto-plastic analysis of pipes. Comparisons with full shell- and beam-type models are provided in order to illustrate the efficiency of the proposed analysis.

The paper is organised as follows; the kinematics and the weak form of the equilibrium equations for shell-type analyses are briefly given in Section 2, those for beam-type are given in Section 3. In Section 4, we introduce the proposed multi-scale analysis 
procedure in detail. Section 5 is the verification of the elasto-plastic shell element implemented in this study. Numerical examples are presented in Section 6 and conclusions are drawn in Section 7.

\section{Shell-type analysis}

\subsection{Kinematic assumptions and strains}

Strains of the shell-type analysis are composed of linear strains due to (a) membrane deformations $\hat{\boldsymbol{\varepsilon}}_{m m}$, (b) plate bending deformations $\hat{\boldsymbol{\varepsilon}}_{b}$, and (c) nonlinear components of strains due to membrane and plate bending action $\hat{\boldsymbol{\varepsilon}}_{N}$, i.e.,

$$
\hat{\boldsymbol{\varepsilon}}=\hat{\boldsymbol{\varepsilon}}_{m m}+\hat{\boldsymbol{\varepsilon}}_{b}+\hat{\boldsymbol{\varepsilon}}_{N}=\left\langle\begin{array}{llll}
\hat{\varepsilon}_{x} & \hat{\varepsilon}_{y} & \hat{\gamma}_{x y} & \hat{\gamma}_{m}
\end{array}\right\rangle^{\mathrm{T}}
$$

Figure 1.a shows the $x$ and $y$ axes defining a plane tangential to the mid-surface of the shell and $z$ axis is normal to the mid-surface. The vector of linear components for the membrane strain $\hat{\boldsymbol{\varepsilon}}_{m}$ can be written as

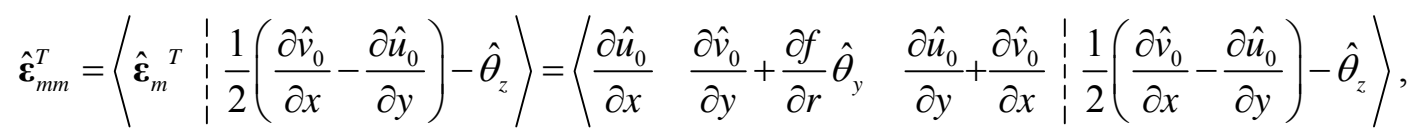

in which $\hat{\theta}_{x}$ and $\hat{\theta}_{y}$ are rotations in local $x-z$ and $y-z$ planes respectively (Fig. 1a), $\hat{\theta}_{z}$ is the drilling rotation about the $z$ axis, and $\hat{u}_{0}$ and $\hat{v}_{0}$ are the displacements of the midsurface in the local $x-y$ plane (Fig. 1.a). In Eq. (2) the term $(\partial f / \partial r) \hat{\theta}_{y}$ is added according to Marguerre shallow shell theory [39]. As shown in Fig. 1.b, $f=f(r)$ is the expression for the elevation of the arch in Z-Y plane in terms of coordinate $r$. In calculating the element length and locations of the integration points, the arch length was considered. For the membrane component of the shell-type element, the finite element of 
Ibrahimbegovic et al. [40] employing drilling degrees of freedom is adopted herein, so that non-coplanar elements can be easily assembled (Fig. 1.c). The last entry in Eq. (2) contains the skew symmetric part of the membrane strains which is introduced into the potential energy functional related to a penalty term to avoid numerical instability when drilling rotations $\hat{\theta}_{z}$ are used with Allman-type interpolations [41]. According to Mindlin-Reissner theory [42], the plate bending strains can be written as

$$
\hat{\boldsymbol{\varepsilon}}_{b}=-z\langle\hat{\chi}: 0\rangle^{T}=-z\left\langle\frac{\partial \hat{\theta}_{x}}{\partial x} \quad \frac{\partial \hat{\theta}_{y}}{\partial y} \quad \frac{\partial \hat{\theta}_{x}}{\partial y}+\frac{\partial \hat{\theta}_{y}}{\partial x}: 0\right\rangle^{T}
$$

in which $\hat{\chi}$ is the curvature vector. It is assumed that the second order longitudinal displacement derivatives, second order lateral strains and second order shear strains are negligibly small, i.e., $\frac{1}{2}\left[\left(\partial \hat{w}_{0} / \partial y\right)^{2}+\left(\partial \hat{u}_{0} / \partial y\right)^{2}\right] \approx 0,\left(\partial \hat{w}_{0} / \partial x\right)\left(\partial \hat{w}_{0} / \partial y\right) \approx 0$. Thus, the nonlinear strain component can be written as

$$
\hat{\boldsymbol{\varepsilon}}_{N} \approx\left\langle\frac{1}{2}\left(\frac{\partial \hat{w}_{0}}{\partial x}\right)^{2}+\frac{1}{2}\left(\frac{\partial \hat{v}_{0}}{\partial x}\right)^{2} \quad 0 \quad 0 \quad 0: 0\right\rangle^{T}
$$

in which $\hat{w}_{0}$ is the out of plane deflection of the mid-surface in local $z$ direction (Fig.1). It should be noted that the second order strain component is consistent with the second order strains of the beam formulation to be introduced in Section 3, so that the beam solution can be considered as a special case of the shell solution. The consistency between beam and shell strains should be examined when adopting higher order shell formulations, e.g., [43] within the proposed multi-scale analysis framework. 


\subsection{Constitutive relations and stresses}

The shell analysis is elasto-plastic. In this study, for convenience, we apply the one step forward Euler numerical procedure as described in [44]. Initially, the vector of total stresses at any point within the shell is

$$
\hat{\boldsymbol{\sigma}}=\left\langle\begin{array}{lll|l}
\hat{\sigma}_{x} & \hat{\sigma}_{y} & \hat{\tau}_{x y} & \hat{\tau}_{m}
\end{array}\right\rangle^{\mathrm{T}}
$$

Assuming that the whole strain increment is elastic, the stress increment can be written as

$$
\Delta \hat{\boldsymbol{\sigma}}=\hat{\mathbf{E}} \Delta \hat{\boldsymbol{\varepsilon}}
$$

in which the matrix of elastic material properties of the shell element $\hat{\mathbf{E}}$ can be written as

$$
\hat{\mathbf{E}}=\frac{E}{\left(1-v^{2}\right)}\left[\begin{array}{ccc:c}
1 & v & 0 & 0 \\
v & 1 & 0 & 0 \\
0 & 0 & \frac{1-v}{2} & 0 \\
\hdashline 0 & 0 & 0 & \frac{(1-v)}{2}
\end{array}\right]
$$

in which $E$ is Young's modulus and $v$ is the Poisson's ratio. The last diagonal term in Eq. (7) arises from the penalty term introduced into the potential energy functional [41]. Within an incremental iterative solution, the trial stresses are obtained using stress increments. Under plane stress plasticity conditions, i.e. $\hat{\sigma}_{z}=\hat{\tau}_{y z}=\hat{\tau}_{z x}=0$, the von Mises yield criterion is used to determine whether the trial stresses are elastic. According to the forward Euler procedure in [44] since $\varepsilon_{z} \neq 0$, a four-dimensional yield surface $f$ is assumed as

$$
f=\sigma_{e f}-\sigma_{Y}
$$


in which $\sigma_{e f}=\left[\left(\hat{\sigma}_{x}-\sigma_{y}\right)^{2}+\left(\hat{\sigma}_{y}-\sigma_{z}\right)^{2}+\left(\hat{\sigma}_{z}-\sigma_{x}\right)^{2}+6 \hat{\tau}_{x y}{ }^{2}\right]^{1 / 2} / \sqrt{2}$ and $\sigma_{Y}$ is the yield stress limit. When yielding occurs, the relation between the stress increment and the elastic strain increment is re-written in a four-dimensional context as

$$
\Delta \hat{\boldsymbol{\sigma}}_{a}=\left\{\begin{array}{l}
\Delta \hat{\sigma}_{x} \\
\Delta \hat{\sigma}_{y} \\
\Delta \hat{\sigma}_{z} \\
\Delta \hat{\tau}_{x y}
\end{array}\right\}=\frac{E}{(1+v)(1-2 v)}\left[\begin{array}{cccc}
(1-v) & v & v & 0 \\
v & (1-v) & v & 0 \\
v & v & (1-v) & 0 \\
0 & 0 & 0 & \frac{1}{2}(1-2 v)
\end{array}\right]\left\{\begin{array}{c}
\Delta \hat{\varepsilon}_{e x} \\
\Delta \hat{\varepsilon}_{e y} \\
\Delta \hat{\varepsilon}_{e z} \\
\Delta \hat{\gamma}_{e x y}
\end{array}\right\}=\mathbf{E}_{a}{ }^{\mathbf{e}} \Delta \boldsymbol{\varepsilon}_{e a}
$$

The elastic part of the strain increment can be expressed as the plastic strain increment subtracted from the total strain, i.e., $\Delta \hat{\boldsymbol{\varepsilon}}_{e a}=\Delta \hat{\boldsymbol{\varepsilon}}_{a}-\hat{\boldsymbol{\varepsilon}}_{p a}$, where $\Delta \hat{\boldsymbol{\varepsilon}}_{a}{ }^{\mathrm{T}}=\left\langle\begin{array}{llll}\Delta \hat{\varepsilon}_{x} & \Delta \hat{\varepsilon}_{y} & \Delta \hat{\varepsilon}_{z} & \Delta \hat{\gamma}_{x y}\end{array}\right\rangle$. Strains $\Delta \hat{\varepsilon}_{x}, \Delta \hat{\varepsilon}_{y}$ and $\Delta \hat{\gamma}_{x y}$ are obtained from the displacement increments as given in Eqs. (2)-(4). Strain $\Delta \hat{\varepsilon}_{z}$ can be obtained by using the plane stress condition, i.e., $\Delta \sigma_{z}=0$ in Eq. (9) while assuming incompressible plasticity [45] yielding

$$
\Delta \varepsilon_{z}=-\frac{v}{(1-v)}\left(\Delta \varepsilon_{x}+\Delta \varepsilon_{y}\right)-\frac{\Delta \lambda}{2 \sigma_{e f}}\left(\sigma_{x}+\sigma_{y}\right) \frac{(1-2 v)}{(1-v)}
$$

From the Prandtl-Reuss flow rule for associative plasticity, the plastic strain increment vector $\Delta \boldsymbol{\varepsilon}_{p a}$ can be written as

$$
\Delta \boldsymbol{\varepsilon}_{p a}=\Delta \lambda \frac{\partial f}{\partial \boldsymbol{\sigma}_{a}}
$$

in which $\Delta \lambda=\frac{\mathbf{a}_{a}{ }^{\mathrm{T}} \mathbf{E}_{a}{ }^{\mathrm{e}} \Delta \boldsymbol{\varepsilon}_{a}}{\mathbf{a}_{a}{ }^{\mathrm{T}} \mathbf{E}_{a}{ }^{\mathrm{e}} \mathbf{a}_{a}}$. By setting $\sigma_{z}=0$, one obtains $\mathbf{a}_{a}$ as

$$
\mathbf{a}_{a}{ }^{\mathrm{T}}=\frac{\partial f^{\mathrm{T}}}{\partial \boldsymbol{\sigma}}=\frac{1}{2 \sigma_{e f}}\left\langle\left(2 \sigma_{x}-\sigma_{y}\right) \quad\left(2 \sigma_{y}-\sigma_{x}\right) \quad\left(-\sigma_{x}-\sigma_{y}\right) \quad 6 \tau_{x y}\right\rangle
$$


It should be noted that $\Delta \hat{\gamma}_{m}$ is assumed elastic. Updated stresses are collected in Eq. (5).

\subsection{Interpolation Functions}

Classical Kirchhoff plate theory is appropriate for thin plates. However, a four node loworder thin plate element formulation is averted by the $C^{1}$ continuity requirements of the Kirchhoff plate theory. On the other hand, standard four node low-order ReissnerMindlin-type elements experience shear locking in the analysis of thin plates. Therefore, we employ the Discrete Kirchhoff Quadrilateral of Batoz and Tahar [46] to express the bending rotations $\hat{\theta}_{x}$ and $\hat{\theta}_{y}$ in Eq. (3), which starts with the Mindlin-Reissner plate theory, neglects the transverse shear strains, and then explicitly couples lateral displacements and rotation fields of the mid-surface normal line to enforce zero transverse shear strain at selected locations. For the buckling analysis of thin-walled beams, the Discrete Kirchhoff Quadrilateral element was previously adopted by Fafard et al. [47]. Similar to [47], we employ standard linear interpolation for the out of plane deflection $\hat{w}$ in Eq. (4) (Fig. 1.a.). The four-node membrane element with drilling degrees-of-freedom uses Allman-type interpolation functions for the in-plane displacements and the standard bilinear independent drilling rotation [40], and to form a shell element, the membrane element is superimposed onto the plate element. Thus, the displacement fields $\hat{\mathbf{u}}=\left\langle\begin{array}{llllll}\hat{u}_{0} & \hat{v}_{0} & \hat{\theta}_{z} & \hat{w}_{0} & \hat{\theta}_{x} & \hat{\theta}_{y}\end{array}\right\rangle^{\mathrm{T}}$ of the shell element can be related to the nodal displacements $\hat{\mathbf{d}}=\left\langle\begin{array}{llllllllllllll}\hat{u}_{1} & \hat{v}_{1} & \hat{\theta}_{z 1} & \hat{w}_{1} & \hat{\theta}_{x 1} & \hat{\theta}_{y 1} & \hat{u}_{2} & \ldots & \hat{u}_{4} & \hat{v}_{4} & \hat{\theta}_{z 4} & \hat{w}_{4} & \hat{\theta}_{x 4} & \hat{\theta}_{y 4}\end{array}\right\rangle^{\mathrm{T}}$ (where subscripts refer to each of the four end nodes) through

$$
\hat{\mathbf{u}}=\hat{\mathbf{X}} \hat{\mathbf{d}}
$$


In Eq. (13), Matrix $\hat{\mathbf{X}}$ contains the shape functions and their derivatives which is explicitly given in Appendix A.

\subsection{Variational formulation and consistent linearization}

For the shell analysis, the equilibrium equations can be obtained in the variational form as

$$
\delta \hat{\Pi}=\int_{L} \int_{A} \delta \hat{\boldsymbol{\varepsilon}}^{\mathrm{T}} \hat{\boldsymbol{\sigma}} \mathrm{d} A \mathrm{~d} \bar{z}-\delta \hat{\mathbf{d}}^{\mathrm{T}} \hat{\mathbf{f}}=0
$$

in which $\hat{\boldsymbol{\varepsilon}}$ represents the vector of strain components. The virtual work functional of the shell element is modified in order to avoid numerical stability issues with Allman type interpolations of the membrane component as suggested in [40] and thus, the skew symmetric part of the membrane strains and associated drilling rotations are contained in the first term in Eq. (14). In the last term of Eq. (14), $\hat{\mathbf{f}}$ is the external load vector. The first variation of the strain field of the shell element used in Eq. (14) can be expressed as

$$
\delta \hat{\boldsymbol{\varepsilon}}=\hat{\mathbf{S}} \hat{\mathbf{B}} \delta \hat{\mathbf{d}}
$$

where $\hat{\mathbf{B}}$ and $\hat{\mathbf{S}}$ for an element are explicitly given in Appendix B. The incremental equilibrium equations for the shell formulation can be obtained by subtracting the first variation of the modified potential energy in Eq. (14) at two neighbouring equilibrium states and then linearizing the results by omitting the second- and higher-order terms, i.e.

$$
\Delta(\delta \hat{\Pi}) \approx \delta \hat{\mathbf{d}}^{\mathrm{T}} \hat{\mathbf{K}} \Delta \hat{\mathbf{d}}-\delta \hat{\mathbf{d}}^{\mathbf{T}} \Delta \hat{\mathbf{f}}=0
$$

where $\hat{\mathbf{K}}$ is the tangent stiffness matrix of the shell model, i.e.,

$$
\hat{\mathbf{K}}=\int_{L} \int_{A} \hat{\mathbf{B}}^{\mathrm{T}} \hat{\mathbf{S}}^{\mathrm{T}} \hat{\mathbf{E}}_{e p} \hat{\mathbf{S}} \hat{\mathbf{B}} \mathrm{d} A \mathrm{~d} \bar{z}+\int_{L} \hat{\mathbf{M}}_{\sigma} \mathrm{d} \bar{z}
$$


where $\hat{\mathbf{M}}_{\sigma} \Delta \hat{\mathbf{d}}=\int_{A} \delta \hat{\mathbf{B}}^{\mathrm{T}} \hat{\mathbf{S}}^{\mathrm{T}} \hat{\boldsymbol{\sigma}} \mathrm{d} A$ and $\hat{\mathbf{E}}_{e p}$ is the elasto-plastic constitutive matrix given in [40].

\section{Beam-type analysis}

\subsection{Kinematic assumptions, strains and stresses}

In order to simplify the global analysis a beam formulation is used, which is based on the classical kinematic assumptions of the Euler-Bernoulli beam theory. These are: (a) plane section remains plane after deformation; (b) longitudinal axis of the pipe stays perpendicular to the cross-sectional plane after deformation; (c) contour of the crosssection does not deform in its plane; (d) normal stresses within the cross-sectional plane (hoop stresses) are zero. These assumptions imply that the nonzero strains in the pipe strain vector, i.e. $\bar{\varepsilon}$ are due to the axial strains induced by membrane and bending actions, and shear strains induced by torsion only. The beam element strain vector can be written in terms of linear and second order nonlinear terms, i.e. $\overline{\boldsymbol{\varepsilon}}=\overline{\boldsymbol{\varepsilon}}_{L}+\overline{\boldsymbol{\varepsilon}}_{N}$. The linear axial and shear strains $\bar{\varepsilon}_{L}$ and $\bar{\gamma}_{L}$, respectively can be obtained in terms of the derivatives of displacements $\bar{u}, \bar{v}, \bar{w}$ and the angle of twist $\bar{\phi}$ (Fig.1.d) as

$$
\overline{\boldsymbol{\varepsilon}}_{L}=\left\langle\begin{array}{llll}
\bar{\varepsilon}_{L} & 0 & \bar{\gamma}_{L} & 0
\end{array}\right\rangle^{\mathrm{T}}=\overline{\mathbf{S}}_{\overline{\boldsymbol{\chi}}_{L}}
$$

which can be decomposed in terms of a matrix of cross-sectional coordinates, i.e.

$$
\overline{\mathbf{S}}=\left[\begin{array}{cccc}
1 & -\bar{x} & -\bar{y} & 0 \\
0 & 0 & 0 & 0 \\
0 & 0 & 0 & \bar{R} \\
0 & 0 & 0 & 0
\end{array}\right]
$$

and a vector of linear displacement derivatives, i.e. 


$$
\bar{\chi}_{L}^{\mathrm{T}}=\left\langle\bar{w}^{\prime} \quad \bar{u}^{\prime \prime} \quad \bar{v}^{\prime \prime} \quad \bar{\phi}^{\prime}\right\rangle
$$

In Eq. (19), $\bar{x}$ and $\bar{y}$ identifies coordinates of a point on the cross-section, and $\bar{R}$ is the radius of the pipe (Fig. 1.a). In Eq. (20), prime denotes derivative with respect to the axial coordinate $z$, i.e. ()$^{\prime}=\mathrm{d}() / \mathrm{d} z$. The nonlinear strains can be written as

$$
\overline{\boldsymbol{\varepsilon}}_{N}=\left\langle\begin{array}{llll}
\bar{\varepsilon}_{N} & 0 & \bar{\gamma}_{N} & 0
\end{array}\right\rangle^{\mathrm{T}}=\overline{\mathbf{S}} \overline{\boldsymbol{\chi}}_{N}
$$

in which $\bar{\varepsilon}_{N}$ is the nonlinear axial strain and $\bar{\gamma}_{N}$ is considered to vanish. Similar to linear strains, the nonlinear strain vector in Eq. (21) can be expressed by using the same matrix of cross-sectional coordinates $\overline{\mathbf{S}}$ post-multiplied by a vector of second-order displacement derivatives, i.e.,

$$
\bar{\chi}_{N}{ }^{\mathrm{T}}=\left\langle\frac{1}{2}\left(\bar{u}^{\prime 2}+\bar{v}^{2}\right) \quad 0 \quad 00 \quad 0 \quad 0\right\rangle .
$$

\subsection{Interpolation functions for the beam displacements}

The element is developed by using linear interpolations for $\bar{w}$ and $\bar{\phi}$ and cubic interpolations for $\bar{u}$ and $\bar{v}$. Thus, the displacement vector of the beam axis is $\overline{\mathbf{u}}_{a}=\overline{\mathbf{X}}_{a} \overline{\mathbf{d}}$, in which

$$
\overline{\mathbf{u}}_{a}=\left\langle\begin{array}{llll}
\bar{w} & \bar{u} & \bar{v} & \bar{\phi}
\end{array}\right\rangle^{\mathrm{T}}
$$

where the matrix of interpolation functions can be written as

$$
\overline{\mathbf{X}}_{a}=\left[\begin{array}{c:c:c:c}
\mathbf{L}^{\mathrm{T}} & \mathbf{0} & \mathbf{0} & \mathbf{0} \\
\hdashline \mathbf{0} & \mathbf{H}^{\mathrm{T}} & \mathbf{0} & \mathbf{0} \\
\hdashline \mathbf{0} & \mathbf{0} & \mathbf{H}^{\mathrm{T}} & \mathbf{0} \\
\hdashline \mathbf{0} & \mathbf{0} & \mathbf{0} & \mathbf{L}^{\mathrm{T}}
\end{array}\right],
$$


In Eq. (24), vectors $\mathbf{L}$ and $\mathbf{H}$ are used for linear and cubic interpolation, respectively, i.e.,

$$
\mathbf{L}=\left\langle\begin{array}{cc}
1-\frac{\bar{Z}}{L} & \frac{\bar{Z}}{L}
\end{array}\right\rangle^{\mathrm{T}}
$$

and

$$
\mathbf{H}=\left\langle 1-\frac{3 \bar{z}^{2}}{L^{2}}+\frac{2 \bar{z}^{3}}{L^{3}} \quad \bar{z}-\frac{2 \bar{z}^{2}}{L}+\frac{\bar{z}^{3}}{L^{2}} \quad \frac{3 \bar{z}^{2}}{L^{2}}-\frac{2 \bar{z}^{3}}{L^{3}} \quad-\frac{\bar{z}^{2}}{L}+\frac{\bar{z}^{3}}{L^{2}}\right\rangle^{\mathrm{T}}
$$

The nodal displacement vector $\overline{\mathbf{d}}$ of the beam type finite element can be written as

$$
\overline{\mathbf{d}}=\left\langle\begin{array}{ll:llll:llll:ll}
\bar{w}_{1} & \bar{w}_{2} & \bar{u}_{1} & \bar{\theta}_{x 1} & \bar{u}_{2} & \bar{\theta}_{x 2} & \bar{v}_{1} & \bar{\theta}_{y 1} & \bar{v}_{2} & \bar{\theta}_{y 2} & \bar{\phi}_{1} & \bar{\phi}_{2}
\end{array}\right\rangle^{\mathrm{T}},
$$

in which subscripts 1 and 2 refer to each of the two end nodes, $\bar{\theta}_{x}$ and $\bar{\theta}_{y}$ refer to bending rotations in $\bar{z}-\bar{x}$ and $\bar{z}-\bar{y}$ planes (Fig.1.d) respectively. Under the rigid sectional contour assumption of the beam theory, the displacement vector of a point $\alpha$ on the cross-section can be written as $\overline{\mathbf{u}}=\mathbf{N} \overline{\mathbf{d}}$ where

$$
\overline{\mathbf{u}}=\left\langle\begin{array}{llllll}
\bar{w}_{\alpha} & \bar{u}_{\alpha} & \bar{u}_{\alpha}^{\prime} & \bar{v}_{\alpha} & \bar{v}_{\alpha} & \bar{\phi}_{\alpha}
\end{array}\right\rangle^{\mathrm{T}},
$$

and $\mathbf{N}=\mathbf{Y Z}$ in which $\mathbf{Z}$ is a matrix of interpolation functions, i.e., 


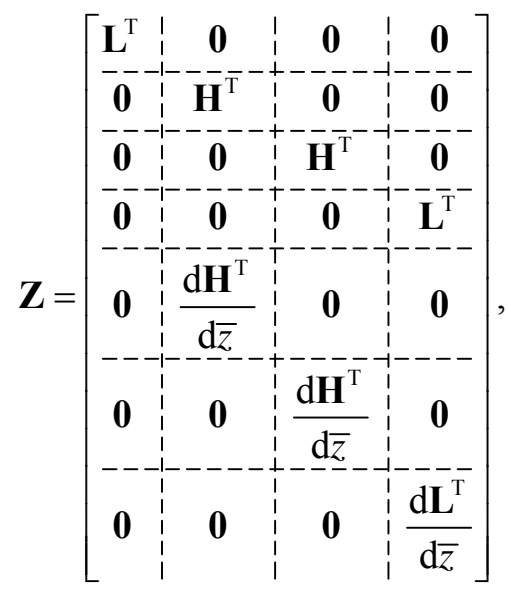

and $\mathbf{Y}$ is a matrix of cross-sectional coordinates, i.e.,

$$
\mathbf{Y}=\left[\begin{array}{ccccccc}
1 & 0 & 0 & 0 & -\bar{x} & -\bar{y} & 0 \\
0 & 1 & 0 & -\bar{y} & 0 & 0 & 0 \\
0 & 0 & 0 & 0 & 1 & 0 & -\bar{y} \\
0 & 0 & 1 & \bar{x} & 0 & 0 & 0 \\
0 & 0 & 0 & 0 & 0 & -1 & -\bar{x} \\
0 & 0 & 0 & -1 & 0 & 0 & 0
\end{array}\right] .
$$

\subsection{Variational formulation and consistent linearization}

The equilibrium equations for static analysis can be obtained in the variational form as

$$
\delta \bar{\Pi}=\iint_{L} \delta \overline{\boldsymbol{\varepsilon}}^{\mathrm{T}} \overline{\boldsymbol{\sigma}} \mathrm{d} A \mathrm{~d} \bar{z}-\delta \overline{\mathbf{d}}^{\mathbf{T}} \overline{\mathbf{f}}=0
$$

in which $A$ is the cross-sectional area, $L$ is the beam span and $\overline{\mathbf{f}}$ is the external load vector. In this study, in the region where no local deformations occur, the material behaviour is assumed elastic. Thus, in Eq. (31), the beam stresses can be obtained directly from the strains using the linear stress-strain relationship for an isotropic material, i.e., $\overline{\boldsymbol{\sigma}}=\overline{\mathbf{E}} \overline{\boldsymbol{\varepsilon}}$, where the vector of beam stresses can be written as

$$
\overline{\boldsymbol{\sigma}}=\left\langle\begin{array}{llll}
\bar{\sigma} & 0 & \bar{\tau} & 0
\end{array}\right\rangle^{\mathrm{T}},
$$


and the beam constitutive matrix $\overline{\mathbf{E}}$ can be written as

$$
\overline{\mathbf{E}}=\left[\begin{array}{ccc:c}
E & 0 & 0 & 0 \\
0 & 0 & 0 & 0 \\
0 & 0 & \frac{E}{2(1+v)} & 0 \\
\hdashline 0 & 0 & 0 & 0
\end{array}\right]
$$

The first variation of the strain vector for the beam element can be written as

$$
\delta \overline{\boldsymbol{\varepsilon}}=\overline{\mathbf{S}} \overline{\mathbf{B}} \delta \overline{\mathbf{d}},
$$

where for an element $\overline{\mathbf{B}}$ can be written as

$$
\overline{\mathbf{B}}=\left[\begin{array}{ccccccc}
1 & \bar{u}^{\prime} & 0 & \bar{v}^{\prime} & 0 & 0 & 0 \\
0 & 0 & 1 & 0 & 0 & 0 & 0 \\
0 & 0 & 0 & 0 & 1 & 0 & 0 \\
0 & 0 & 0 & 0 & 0 & 0 & 1
\end{array}\right] \nabla \overline{\mathbf{X}}_{a}
$$

in which

$$
\nabla=\left[\begin{array}{ccccccc}
\frac{\mathrm{d}}{\mathrm{d} z} & 0 & 0 & 0 & 0 & 0 & 0 \\
0 & \frac{\mathrm{d}}{\mathrm{d} z} & \frac{\mathrm{d}^{2}}{\mathrm{~d} z^{2}} & 0 & 0 & 0 & 0 \\
0 & 0 & 0 & \frac{\mathrm{d}}{\mathrm{d} z} & \frac{\mathrm{d}^{2}}{\mathrm{~d} z^{2}} & 0 & 0 \\
0 & 0 & 0 & 0 & 0 & 1 & \frac{\mathrm{d}}{\mathrm{d} z}
\end{array}\right]^{\mathrm{T}}
$$

The incremental equilibrium equations can be obtained by subtracting the virtual work expressions at two neighbouring equilibrium states and then linearising the result by omitting the second- and higher-order terms, i.e.,

$$
\Delta(\delta \bar{\Pi}) \approx \delta \overline{\mathbf{d}}^{\mathrm{T}} \overline{\mathbf{K}} \Delta \overline{\mathbf{d}}-\delta \overline{\mathbf{d}}^{\mathrm{T}} \Delta \overline{\mathbf{f}}=0
$$

where $\overline{\mathbf{K}}$ is the stiffness matrix of the global beam model, i.e., 


$$
\overline{\mathbf{K}}=\int_{L} \int_{A} \overline{\mathbf{B}}^{\mathrm{T}} \overline{\mathbf{S}}^{\mathrm{T}} \overline{\mathbf{E}} \overline{\mathbf{S}} \overline{\mathbf{B}} \mathrm{d} A \mathrm{~d} \bar{z}+\int_{L} \overline{\mathbf{M}}_{\sigma} \mathrm{d} \bar{z}
$$

in which $\overline{\mathbf{M}}_{\sigma} \Delta \overline{\mathbf{d}}=\delta \overline{\mathbf{B}}^{\mathrm{T}} \int_{A} \overline{\mathbf{S}}^{\mathrm{T}} \overline{\boldsymbol{\sigma}} \mathrm{d} A$.

\section{Multi-scale analysis procedure}

\subsection{Overlapping multi-scale domain decomposition:}

Proposed multi-scale analysis is performed only in a critical part of the analysis domain depicted as $\Omega_{m}$ in Figure 2. In the multi-scale analysis domain, the beam and shell models overlap. The whole analysis domain including sub-domain $\Omega_{m}$ is represented with $\Omega_{c}$ in Figure 2. The beam model is used for the whole analysis domain $\Omega_{c}$. Following the Bridging multi-scale approach of Liu and his co-workers [24-28], we decompose the shell nodal displacement vector $\hat{\mathbf{d}}$ into a coarse-scale component and a difference term, by using a decomposition matrix $\mathbf{N}$ that projects the beam solution onto the nodal points of the shell model, i.e.,

$$
\delta \hat{\mathbf{d}}=\mathbf{N} \delta \overline{\mathbf{d}}+\delta \mathbf{d}^{\prime},
$$

in which the first term on the right-hand side represents the variation of the coarse-scale solution and the second term represents the difference from the fine-scale solution. The decomposition matrix $\mathbf{N}$ constrains the shell model to behave as a beam and thus the strain vector $\overline{\boldsymbol{\varepsilon}}$ of the beam formulation can be obtained by using the displacement field $\overline{\mathbf{u}}=\mathbf{N} \overline{\mathbf{d}}$ in the shell strain derivations $\hat{\boldsymbol{\varepsilon}}$ in Eq. (1). By adopting $\mathbf{N}$ and thus, imposing beam kinematics on the shell model, we define a unique coarse-scale decomposition of the shell solution. In this case, the variation of the coarse scale strain component of the shell solution, i.e. $\delta \overline{\boldsymbol{\varepsilon}}$ is equal to that of the beam, i.e. $\hat{\mathbf{S}} \hat{\mathbf{B}} \mathbf{N}=\overline{\mathbf{S}} \overline{\mathbf{B}}$. 


\subsection{Coupled coarse- and fine-scale equilibrium equations}

Based on the above decomposition, by substituting Eq. (39) into Eq. (15), i.e., $\delta \hat{\boldsymbol{\varepsilon}}=\hat{\mathbf{S}} \hat{\mathbf{B}}\left(\mathbf{N} \delta \overline{\mathbf{d}}+\delta \mathbf{d}^{\prime}\right)$, the first variation of the shell strains can be decomposed into two components, i.e., $\delta \hat{\boldsymbol{\varepsilon}}=\delta \overline{\boldsymbol{\varepsilon}}+\delta \boldsymbol{\varepsilon}^{\prime}$ as

$$
\delta \overline{\boldsymbol{\varepsilon}}=\hat{\mathbf{S}} \hat{\mathbf{B}} \mathbf{N} \delta \overline{\mathbf{d}}
$$

and

$$
\delta \boldsymbol{\varepsilon}^{\prime}=\hat{\mathbf{S}} \hat{\mathbf{B}} \delta \mathbf{d}^{\prime}
$$

In Eq. (41), $\delta \boldsymbol{\varepsilon}^{\prime}$ is due to the difference between the variations of the fine and coarsescale strain fields. The stress fields are also decomposed into two components i.e. $\hat{\boldsymbol{\sigma}}=\overline{\boldsymbol{\sigma}}+\boldsymbol{\sigma}^{\prime}$. By considering the constitutive relations, the stress field components can be obtained from the associated strain fields. From Eqs. (39)-(41), by substituting into Eq. (14), the weak form of the shell equilibrium equations can be decomposed into two simultaneous equations, i.e.,

$$
\delta \Pi_{1}=\delta \overline{\mathbf{d}}^{\mathrm{T}} \mathbf{N}^{\mathrm{T}} \int_{L} \int_{A} \hat{\mathbf{B}}^{\mathrm{T}} \hat{\mathbf{S}}^{\mathrm{T}} \overline{\boldsymbol{\sigma}} \mathrm{d} A \mathrm{~d} \bar{z}-\delta \overline{\mathbf{d}}^{\mathrm{T}} \mathbf{N}^{\mathrm{T}} \hat{\mathbf{f}}+\delta \overline{\mathbf{d}}^{\mathrm{T}} \mathbf{F}=0
$$

and

$$
\delta \Pi_{2}=\delta \mathbf{d}^{\prime \mathrm{T}} \int_{L} \int_{A} \hat{\mathbf{B}}^{\mathrm{T}} \hat{\mathbf{S}}^{\mathrm{T}} \hat{\boldsymbol{\sigma}} \mathrm{d} A \mathrm{~d} \overline{\bar{z}}-\delta \mathbf{d}^{\prime \mathrm{T}} \hat{\mathbf{f}}=0
$$

It is observed that starting with the equilibrium condition for the shell analysis (Eq.(14))

and substituting Eq. (15), one obtains $\delta \widehat{\Pi}=\delta \hat{\mathbf{d}}^{\mathrm{T}}\left(\iint_{L} \hat{\mathbf{B}}_{A}^{\mathrm{T}} \hat{\mathbf{S}}^{\mathrm{T}} \hat{\boldsymbol{\sigma}} \mathrm{d} A \mathrm{~d} \bar{z}-\hat{\mathbf{f}}\right)=0$. Since $\delta \hat{\mathbf{d}}$ is arbitrary, it can be replaced by $\delta \mathbf{d}^{\prime \mathbf{T}}$ yielding Eq. (43). It is also noted that within the 
Bridging multi-scale framework, the coarse- and fine-scale decomposition is applied to the discrete nodal values, and thus the relation $\iint_{L} \mathbf{N}_{A}^{\mathrm{T}} \hat{\mathbf{B}}^{\mathrm{T}} \hat{\mathbf{S}}^{\mathrm{T}} \boldsymbol{\sigma d} A \mathrm{~d} \bar{z}=\mathbf{N}^{\mathrm{T}} \int_{L} \int_{A} \hat{\mathbf{B}}^{\mathrm{T}} \hat{\mathbf{S}}^{\mathrm{T}} \boldsymbol{\sigma d} A \mathrm{~d} \bar{z}$ holds. Accordingly, it can be shown that the first term in Eq. (31) satisfies the identity $\int_{L} \int_{A} \delta \overline{\boldsymbol{\varepsilon}}^{\mathrm{T}} \overline{\boldsymbol{\sigma}} \mathrm{d} A \mathrm{~d} \bar{Z}=\delta \overline{\mathbf{d}}^{\mathrm{T}} \mathbf{N}^{\mathrm{T}} \iint_{L} \hat{\mathbf{B}}_{A}^{\mathrm{T}} \hat{\mathbf{S}}^{\mathrm{T}} \overline{\boldsymbol{\sigma}} \mathrm{d} A \mathrm{~d} \bar{Z}$. For a loading case where $\mathbf{N}^{\mathrm{T}} \hat{\mathbf{f}}=\overline{\mathbf{f}}$ (i.e., the same loading is applied to the fine and coarse-scale analyses), one can show that the second term of Eq. (31) satisfies the identity $\delta \overline{\mathbf{d}}^{\mathrm{T}} \overline{\mathbf{f}}=\delta \overline{\mathbf{d}}^{\mathrm{T}} \mathbf{N}^{\mathrm{T}} \hat{\mathbf{f}}$. By using Eqs. (31) and (43), one obtains Eq. (42) from the relation $\delta \Pi_{1}=\delta \hat{\Pi}-\delta \Pi_{2}=0$. What separates the equilibrium condition for the beam as given in Eq. (31) from that of Eq. (42) is the presence of the last term $\delta \overline{\mathbf{d}}^{\mathrm{T}} \mathbf{F}$, in which $\mathbf{F}$ is a complementary force vector due to fine and coarse-scale differences in the stress field, expressed as

$$
\mathbf{F}=\mathbf{N}^{\mathrm{T}} \int_{L} \int_{A} \hat{\mathbf{B}}^{\mathrm{T}} \hat{\mathbf{S}}^{\mathrm{T}}(\hat{\boldsymbol{\sigma}}-\overline{\boldsymbol{\sigma}}) \mathrm{d} A \mathrm{~d} \bar{Z}=\int_{L} \int_{A} \overline{\mathbf{B}}^{\mathrm{T}} \overline{\mathbf{S}}^{\mathrm{T}} \boldsymbol{\sigma}^{\prime} \mathrm{d} A \mathrm{~d} \bar{Z}
$$

\subsection{Linearization of the equilibrium equations}

Linearization of Eq. (42) produces

$$
\Delta\left(\delta \Pi_{1}\right) \approx \delta \overline{\mathbf{d}}^{\mathbf{T}} \overline{\mathbf{K}} \Delta \overline{\mathbf{d}}-\delta \overline{\mathbf{d}}^{\mathbf{T}} \Delta \overline{\mathbf{f}}=0,
$$

where $\overline{\mathbf{K}}=\mathbf{N}^{\mathrm{T}}\left(\iint_{L} \hat{\mathbf{B}}^{\mathrm{T}} \hat{\mathbf{S}}^{\mathrm{T}} \overline{\mathbf{E}} \hat{\mathbf{S}} \hat{\mathbf{B}} \mathrm{d} A \mathrm{~d} \bar{z}+\int_{L} \tilde{\mathbf{M}}_{\sigma} \mathrm{d} \bar{z}\right) \mathbf{N}$ and can be replaced with the beam stiffness matrix in Eq. (38), in which $\tilde{\mathbf{M}}_{\sigma}$ is defined as $\tilde{\mathbf{M}}_{\sigma} \Delta \hat{\mathbf{d}}=\int_{A} \delta \hat{\mathbf{B}}^{\mathrm{T}} \hat{\mathbf{S}}^{\mathrm{T}} \overline{\mathbf{E}} \hat{\boldsymbol{\varepsilon}} \mathrm{d} A$. In obtaining Eq. (45), the variation of the difference between nodal displacements of fineand coarse-scales is decomposed as $\delta \mathbf{d}^{\prime}=\mathbf{N} \delta \mathbf{c}+\mathbf{Q} \delta \mathbf{q}$, in which $\delta \mathbf{c}$ can be selected as

$$
\delta \mathbf{c}=-\left[\mathbf{N}^{\mathrm{T}} \hat{\mathbf{K}} \mathbf{N}\right]^{-1} \mathbf{N}^{\mathrm{T}} \int_{L} \int_{A}\left[\hat{\mathbf{B}}^{\mathrm{T}} \hat{\mathbf{S}}^{\mathrm{T}}(\hat{\mathbf{E}}-\overline{\mathbf{E}}) \hat{\mathbf{S}} \hat{\mathbf{B}}+\mathbf{k}_{\sigma}\right] \mathrm{d} A \mathrm{~d} \overline{\mathbf{Z}} \mathbf{N} \delta \overline{\mathbf{d}},
$$


in which $\mathbf{k}_{\sigma} \delta \hat{\mathbf{d}}=\delta \hat{\mathbf{B}}^{\mathrm{T}} \hat{\mathbf{S}}^{\mathrm{T}}(\hat{\mathbf{E}}-\overline{\mathbf{E}}) \hat{\mathbf{\varepsilon}}$ and matrix $\mathbf{Q}$ can be selected as [26],

$$
\mathbf{Q}=\mathbf{I}-\mathbf{N}\left[\mathbf{N}^{\mathrm{T}} \hat{\mathbf{K}} \mathbf{N}\right]^{-1} \mathbf{N}^{\mathrm{T}} \hat{\mathbf{K}}
$$

so that the orthogonality relation between $\mathbf{N}$ and $\mathbf{Q}$ is satisfied for an arbitrary nodal vector of displacements $\delta \mathbf{q}$, i.e. $\mathbf{N}^{\mathrm{T}} \hat{\mathbf{K}} \mathbf{Q}=0$ in which $\hat{\mathbf{K}}$ is as in Eq. (17).

On the other hand, linearization of Eq. (43) produces;

$$
\Delta\left(\delta \Pi_{2}\right) \approx \delta \mathbf{d}^{\prime \mathbf{T}} \hat{\mathbf{K}} \Delta \hat{\mathbf{d}}-\delta \mathbf{d}^{\prime \mathbf{T}} \Delta \hat{\mathbf{f}}=0 .
$$

Since $\delta \mathbf{d}^{\prime}$ in Eq. (48) is arbitrary, both Eq. (16) and Eq. (48) admit the same solution, which is the solution of the shell model on the entire analysis domain. However, where the beam solution is accurate enough, the shell model solution is avoided for economy.

\subsection{Interface boundary conditions and partitioning of the linearized fine-scale equations}

In obtaining the shell solution, we deviate from the original applications of the Bridging multi-scale method, e.g. [25-28] in which the coarse-scale solution within the overlapping domain is used to obtain the difference between the fine- and coarse-scale displacements. Instead, it is more convenient to obtain the shell solution within the overlapping domain by imposing the displacements of the beam solution as the interface boundary conditions of the shell model. An important issue to be addressed before imposing the shell boundary conditions is that even though there are no local buckling deformations, the Poisson ratio effect causes change in the cross-sectional contour dimensions throughout the analysis domain. However, beam analysis does not produce a displacement field within the plane of the cross-section that captures the changes in cross-sectional dimensions due to Poisson ratio effect. Thus, it is required to consider the changes in the 
cross-sectional contour dimensions before imposing shell model boundary conditions at the interface of the multi-scale region ( $\partial \Omega_{s}$ in Fig.2). At both boundaries of the shell model, fine scale displacement vector due to Poisson ratio effect, i.e. $\tilde{\mathbf{d}}_{B}$ (where subscript B denotes the boundaries $i \& j$ at both ends of the local shell model as shown in Fig. 2) is obtained by numerically integrating the hoop strains $-v \varepsilon$ over the cross-sectional contour, and then setting the summation of these displacements to zero in order to eliminate the rigid body translations due to $\tilde{\mathbf{d}}_{B}$. Thus, the displacement boundary conditions imposed onto the shell model can be written as $\hat{\mathbf{d}}_{B} \approx \mathbf{N} \overline{\mathbf{d}}_{B}+\tilde{\mathbf{d}}_{B}$. From Eq. (48), by decomposing the shell displacement vector into boundary and internal displacement vectors, one obtains

$$
\underbrace{\left[\begin{array}{c:c}
\hat{\mathbf{K}}_{a} & \hat{\mathbf{K}}_{b} \\
\hdashline \hat{\mathbf{K}}_{b}^{\mathrm{T}} & \hat{\mathbf{K}}_{c}
\end{array}\right]}_{\hat{\mathbf{K}}} \underbrace{\left\{\begin{array}{c}
\Delta \hat{\mathbf{d}}_{B} \\
-\Delta \hat{\mathbf{d}}_{I N}
\end{array}\right\}}_{\Delta \hat{\mathbf{d}}}=\underbrace{\left\{\begin{array}{c}
\Delta \hat{\mathbf{f}}_{B} \\
-- \\
\Delta \hat{\mathbf{f}}_{s}
\end{array}\right\}}_{\Delta \hat{\mathbf{f}}}
$$

The stiffness matrix $\hat{\mathbf{K}}$ of the shell model in Eq. (17), is partitioned such that specified boundary displacements are multiplied with sub-matrix $\hat{\mathbf{K}}_{b}{ }^{\mathrm{T}}$. In Eq. (49), $\Delta \hat{\mathbf{f}}_{s}$ is the vector of increment in specified external loads that fall into the multi-scale analysis domain and $\Delta \hat{\mathbf{f}}_{B}$ is the vector of increment in traction forces at the boundaries of the multi-scale analysis domain. Specified displacements and loads in Eq. (49) are placed in the box symbol $(\square)$.

\subsection{Solution procedure for the nonlinear equilibrium equations}

Firstly, the global problem is solved for the coarse-scale displacements $\overline{\mathbf{d}}$ while keeping the fine-scale solution of the local shell model fixed. Then, given the global results imposed on the local model as the interface boundary conditions, the local problem is 
solved for the fine-scale values $\hat{\mathbf{d}}$, while keeping the boundary conditions and the global displacements $\overline{\mathbf{d}}$ fixed. In order to terminate the loading step $k$, we use double criteria as suggested in Qian et al [26] within the framework of Bridging multiscale method. The first criterion is due to geometric nonlinearity and confirms that the nonlinear global equilibrium condition is satisfied at the end of $n$ iterations. A second criterion is required to confirm that the difference in the stress vectors of the local shell model and the beam model is eliminated through the complementary force in Eq. (44), thus synchronizing the local and global solutions. Within the multi-scale analysis scheme developed herein, the span of the overlapping domain can be adjusted at any load level, because the local shell model is solved for the current loading conditions at the beginning of each step, regardless of the results of the shell model obtained in the previous steps. A flow chart of the solution procedure is given in Fig. 3 .

The global equations are solved using a Newton-Raphson incremental-iterative scheme in a step-by-step manner, i.e.

$$
\overline{\mathbf{K}}_{k} \Delta \overline{\mathbf{d}}_{k}^{n}=\Delta \overline{\mathbf{f}}_{k}+\Delta \overline{\mathbf{R}}_{k}^{n}
$$

where $\overline{\mathbf{K}}_{k}$ is the tangent stiffness matrix at the beginning of each incremental step, $\Delta \overline{\mathbf{f}}_{k}$ is the external load increment in step $k$, and $\Delta \overline{\mathbf{R}}_{k}^{n}$ is the unbalanced force vector obtained from Eq. (42) at the $n^{\text {th }}$ iteration of step $k$, i.e.

$$
\Delta \overline{\mathbf{R}}_{k}^{n}=-\int_{L} \int_{A} \overline{\mathbf{B}}_{k}^{n \mathrm{~T}} \overline{\mathbf{S}}^{\mathrm{T}} \overline{\boldsymbol{\sigma}}_{k}^{n} \mathrm{~d} A \mathrm{~d} \bar{z}-\mathbf{F}_{k}^{n}+\overline{\mathbf{f}}_{k}
$$

The incremental nodal displacements obtained from Eq. (50) are used in updating the displacement configuration of the current state, i.e. $\overline{\mathbf{d}}_{k}^{n}=\overline{\mathbf{d}}_{k}^{n-1}+\Delta \overline{\mathbf{d}}_{k}^{n}$, based on which the 
internal strain field $\overline{\boldsymbol{\varepsilon}}_{k}^{n}$ and consequently the stress field $\overline{\boldsymbol{\sigma}}_{k}^{n}$ of the coarse-scale solution can be updated. Within the load step $k$, fine-scale displacements can be obtained again in an incremental-iterative step-by-step manner; firstly, displacement boundary conditions and the load within the global step $k$, are imposed in $s$ steps and; secondly, the displacement increments of the internal nodes are determined in $l$ iterations, i.e. $\Delta \hat{\mathbf{d}}_{I N}{ }_{s}^{l}$, because the unbalanced terms due to the geometric nonlinearities, i.e. $\Delta \hat{\mathbf{r}}_{I N}{ }_{s}^{l}$ should also be corrected in the local model. Thus, from the second line of Eq. (49), this fine-scale displacement vector of the internal nodes at the $l^{\text {th }}$ iteration $\Delta \hat{\mathbf{d}}_{I N}{ }_{s}^{l}$ can be written as

$$
\Delta \hat{\mathbf{d}}_{I N}{ }^{l}=\hat{\mathbf{K}}_{c}{ }^{-1}\left(\Delta \hat{\mathbf{f}}_{s}+\Delta \hat{\mathbf{r}}_{I N}{ }_{s}^{l}-\hat{\mathbf{K}}_{b}{ }^{\mathrm{T}} \Delta \hat{\mathbf{d}}_{s B}\right)
$$

in which $\Delta \hat{\mathbf{d}}_{s B}$ indicates the specified displacement increments at the boundaries of the fine-scale domain. In Eq. (52), $\Delta \hat{\mathbf{r}}_{I N}^{l}$ is the unbalanced load vector due to geometric nonlinearities involved in the local shell problem, which can be obtained as

$$
\Delta \hat{\mathbf{r}}_{s}^{l}=-\int_{L} \int_{A} \hat{\mathbf{B}}_{s}^{l \mathrm{~T}} \hat{\mathbf{S}}^{\mathrm{T}} \hat{\boldsymbol{\sigma}}_{s}^{l} \mathrm{~d} A \mathrm{~d} \bar{z}+\hat{\mathbf{f}}_{s}
$$

The incremental shell nodal displacements obtained from Eq. (52) are used in updating the displacement configuration of the current state, i.e. $\hat{\mathbf{d}}_{s}^{l}=\hat{\mathbf{d}}_{s}^{l-1}+\Delta \hat{\mathbf{d}}_{s}^{l}$, based on which the internal strain field $\hat{\boldsymbol{\varepsilon}}_{s}^{l}$ and consequently the stress field $\hat{\boldsymbol{\sigma}}_{s}^{l}$ of the fine-scale solution can be updated. If the local convergence criterion is satisfied, i.e. $\left\|\Delta \hat{\mathbf{r}}_{s}^{l}\right\|<\varepsilon_{\text {tol }}$ then $\hat{\boldsymbol{\sigma}}_{k}^{n}=\hat{\boldsymbol{\sigma}}_{s}^{l}$ is used for the complementary force calculations within the $k^{\text {th }}$ step of the $n^{\text {th }}$ iteration, i.e. $\mathbf{F}_{k}^{n}=\mathbf{N}^{\mathrm{T}} \int_{L} \int_{A} \hat{\mathbf{B}}_{k}^{n \mathrm{~T}} \hat{\mathbf{S}}^{\mathrm{T}} \hat{\boldsymbol{\sigma}}_{k}^{n} \mathrm{~d} A \mathrm{~d} \bar{z}-\int_{L} \int_{A} \overline{\mathbf{B}}_{k}^{n \mathrm{~T}} \overline{\mathbf{S}}^{\mathrm{T}} \overline{\boldsymbol{\sigma}}_{k}^{n} \mathrm{~d} A \mathrm{~d} \bar{z}$. It should be noted that for a stable global system the global equilibrium should be satisfied for any complementary force vector. 
Therefore, even though we reach the convergence criterion for global equations, i.e. $\left\|\Delta \overline{\mathbf{R}}_{k}^{n}\right\|<\varepsilon_{\text {tol }}$, step $k$ deemed complete only when $\left\|\Delta \mathbf{F}_{k}^{n}\right\|<\varepsilon_{\text {tol }}$ is satisfied, so that the local and global solutions are synchronized. If both convergence criteria are not satisfied, the analysis should be repeated for a reduced load increment within the same step $k$. Within the multi-scale analysis scheme developed herein, the span of the overlapping domain can be adjusted at any load level, because the local shell model is solved for the current loading conditions at the beginning of each step, regardless of the results of the shell model obtained in the previous steps.

\section{Verification of shell analysis}

Before using multi-scale developments in the present model, the elasto-plastic shell model implemented in Section 2 was verified. Towards this goal, a cylinder panel under point load was considered. As shown in Fig. 4.a, the curve edge nodes of the panel are assumed to be free in all directions while the side nodes are fixed against translation in all three directions. The modulus of elasticity, the Poisson ratio and the yield stress is taken as $3.103 \mathrm{kN} / \mathrm{mm}^{2}, 0.3$, and $.001 \mathrm{kN} / \mathrm{mm}^{2}$, respectively. The results are obtained by using 20x10 elements, i.e., 20 elements along the curved direction and 10 elements along the fixed edge direction, and compared with those obtained by the TRIC continuum formulation of Argyris et al [48] as shown in Fig. 4.b and excellent agreement is observed

\section{Numerical examples}

\subsection{Cantilever pipe under compression, lateral force, and pinching forces}

As shown in Fig. 5.a, the pipe analysed has a $17.15 \mathrm{~m}$ span, a $1 \mathrm{~m}$ diameter and a $25 \mathrm{~mm}$ wall thickness. The pipe is fixed at the bottom end and is subject to a compressive force up to $N=15000 \mathrm{kN}$ acting at the top and a total lateral load up to $15 \mathrm{kN}\left(F_{Y}=7.5 \mathrm{kN}\right)$. The 
pipe is pinched at height $\bar{Z}=3430 \mathrm{~mm}$ through two equal and opposite sets of five forces 5Py as shown in Fig. 5 (up to a value of $P_{Y}=1800 \mathrm{kN}$ ), in order to cause distortional deformations on the cross-section as well as plastic deformations. Modulus of elasticity and the Poisson's ratio used in this example and the next example are $E=200 \times 10^{3} \mathrm{MPA}$ and $v=0.3$, respectively. The yield stress was taken as $300 \mathrm{MPa}$ and no hardening was assumed.

For the beam-type and multi-scale analyses four equal-span elements are used. In the shell analysis, the cross-section was divided into 12 shell elements and the pipe span was sub-divided into 30 elements. The axial load is applied as distributed load acting at the nodes of the cross-section of the shell model. In the multi-scale analysis the cross-section is again divided into 12 shell elements and span was sub-divided into 14 elements. In order to verify the validity of the beam-type analyses, we also present a comparison against the constrained shell solution which is obtained by applying multiple-point constraints on the nodal displacements of the shell model based on the decomposition matrix $\mathbf{N}$ and adopting the beam constitutive matrix $\overline{\mathbf{E}}$.

Firstly, a linearly elastic analysis was conducted. The applied loads were a compressive force (i.e., $N=15000 \mathrm{kN})$ and a small lateral force, $\left(F_{Y}=7.5 \mathrm{kN}\right)$. The load versus tip horizontal deflection and tip rotation curves are plotted as shown in Figs $6 \mathrm{a}$, and $\mathrm{b}$ respectively.

Secondly, buckling loads based on a linearized buckling analysis corresponding to beamtype, constraint-shell-type and full-shell-type analysis are found as $P_{A}=15,277 \mathrm{kN}$, $P_{A}=15,740 \mathrm{kN}$ and $P_{A}=15,587 \mathrm{kN}$, respectively, thus verifying the validity of beam 
analysis model, and suggesting that ovalization in this case have a negligible effect on the results. In the shell analysis, it was verified that no plastic deformations have taken place.

Thirdly, a nonlinear analysis was conducted, in which the loads were incremented from $N=0, F_{y}=0$ to a maximum of $N=15000 \mathrm{kN}, F_{Y}=7.5 \mathrm{kN}$ without pinching loads (i.e., $P_{Y}=0$ ). As shown in Figs. $6 a-b$, excellent agreement is observed between the beam analysis and the shell analysis.

Fourthly, in addition to the applied compressive loads, pinching loads were incrementally applied from zero to $P_{Y}=1800 \mathrm{kN}$ in order to induce additional distortional deformations as well as plastic deformation. The corresponding load versus deflection curves are also shown in Fig. 6.a and b. It should be noted that comparison with fully elastic solution under local loads shows that plastic deformations are attained. The load versus deflection curves for the constrained shell and beam-type solutions are observed to be identical to those of the case where $P_{Y}=0$. On the other hand, when local deformations are introduced, the plastic deformations cause softening effect and increase the overall deflections of the full shell-type solution, which are not captured using the beam-type analysis given the rigid cross-section and elastic material response assumptions. In contrast, the multi-scale solution is very efficient in capturing the same behaviour as that predicted by the full shell-type analysis. In the multi-scale analysis, an overlapping region was considered between $\bar{Z}=0$ and $\bar{Z}=8003.33 \mathrm{~mm}$. The results are in very good agreement as can be verified from Fig. 6. In Fig. 7, the deformed shape and the stress contour for stresses in the longitudinal direction based on full shell-type analysis are shown for the loading of $N=7340 \mathrm{kN}, F_{Y}=3.67 \mathrm{kN}$ and $P_{Y}=880.8 \mathrm{kN}$. In Figs. 8, stresses in longitudinal direction based on shell-type analysis are compared with those of the 
multi-scale solution. The stresses are obtained at the middle of the elements by averaging the stresses at four integration points of the element. It can be verified that the multi-scale analysis results are very close to those of the shell analysis results.

\subsection{Ovalization in a simply supported pipe}

As shown in Fig. 9, a 5m span simply supported horizontal pipe with a $200.25 \mathrm{~mm}$ diameter and 3.25 wall thickness is analyzed. The horizontal pipe is subject to a compressive force up to $N=6000 \mathrm{kN}$. Self -weight of the beam is also considered in the analysis. In order to suppress inelastic behaviour, the yield stress was taken as $1450 \mathrm{MPa}$. For the beam-type and multi-scale analyses, four equal-span elements are used. For the shell analysis the cross-section was divided into 12 elements and the span was divided into 16 elements. In the multi-scale analysis the cross-section is again divided into 12 shell elements and span was sub-divided into 12 elements. Buckling loads based on the linearized buckling analysis corresponding to beam-type and full-shell-type analysis are $P_{A}=6477 \mathrm{kN}$ and $P_{A}=6022 \mathrm{kN}$, respectively.

As shown in Fig. 10, load versus mid-span deflection curves are plotted up to a compressive load of $6000 \mathrm{kN}$. A comparison of the displacements at the top and side nodes suggests the model successfully captures ovalization induced by bending. Results based on the multiscale analysis nearly exactly match those based on the shell solution and thus successfully capture the effects of ovalization. The deformed shape based on shell analysis is shown in Fig. 11 and the stress contours based on full shell-type, full beam-type and multi-scale analyses are shown in Figs. 12. Very good agreement is also observed between the longitudinal stresses predicted by the shell model and that based on the multi-scale model. 


\section{Conclusions}

In this paper, an analysis method based on the Bridging multi-scale approach was developed for the elasto-plastic analysis of pipes. The multi-scale domain decomposition allows the method to incorporate the effects of local deformations on the overall behaviour of the pipe by using a shell model only within the region of local deformations. A pipe buckling case was analysed and the results of the multi-scale analysis procedure proposed herein were compared with those of the full shell- and beam-type analyses. It was shown that very accurate results are obtained using the proposed analysis procedure. Effect of ovalization on the behaviour of a thin-walled pipe is also illustrated and it was shown that ovalization effect was successfully captured by using the multi-scale analysis procedure.

\section{References}

1. Karamanos (2002). Bending instabilities of elastic tubes. International Journal of Solids and Structures 39: 2059-2085.

2. Hobbs RE (1981). Pipeline buckling caused by axial loads. Journal of Constructional Steel Research 1(2): 2-10.

3. Karamanos, S. A. and Tassoulas, J. L. (1996), "Tubular Members I: Stability Analysis and Preliminary Results.", J. Engineering Mechanics, ASCE, Vol. 122, No. 1, pp.64-71.

4. Houliara, S. and Karamanos, S. A. (2006), "Buckling and Post-Buckling of Pressurized Thin-Walled Elastic Tubes Under In-Plane Bending.", International Journal of Nonlinear Mechanics, Vol. 41, No. 4, pp. 491-511.

5. Houliara, S. and Karamanos, S. A. (2010), "Stability of Long TransverselyIsotropic Elastic Cylinders Under Bending.", International Journal of Solids and Structures, Vol. 47, No. 1, pp. 10-24. 
6. Belytschko T, Korungauz Y, Organ D, Fleming M, Krysl P (1996). Meshless methods: An overview and recent developments. Computer Methods in Applied Mechanics and Engineering; 139:3-47.

7. Duarte CAM, Oden JT (1996). Hp clouds - an hp meshless method. Numerical Methods for Partial Differential Equations; 12:673-705.

8. Erkmen RE, Bradford MA (2011). Coupling of finite element and meshfree methods for locking-free analysis of shear deformable beams and plates. Engineering Computations; 28: 1003-1027.

9. Babuska I, Melenk JM (1997). The partition of unity finite element method. International Journal for Numerical Methods in Engineering; 40:727-758.

10. Belytschko T, Moes N, Usui S, Parimi C (2001). Arbitrary discontinuities in finite elements. International Journal for Numerical Methods in Engineering; 50:9931013.

11. Strouboulis T, Copps K, Babuska I (2001). The generalized finite element method. Computer Methods in Applied Mechanics and Engineering; 190: 40814193.

12. Fish J, Markolefas S, Guttal R, Nayak P (1994). On adaptive multilevel superposition of finite element meshes for linear elastostatics. Applied Numerical Mathematics; 14: 135-164.

13. Hughes TJR, Fiejoo G, Mazzei L, Quincy JB (1998). The variational multiscale method- a paradigm for computational mechanics. Computer Methods in Applied Mechanics and Engineering; 166: 3-24.

14. Hughes TJR, Sangalli G (2007). Variational multiscale analysis: The fine-scale Green's function, projection, optimization, localization and stabilized methods. SIAM Journal of Numerical Analysis; 45: 539-557. 
15. Liu WK, Hao S, Belytschko T, Li S, Chang CT (2000). Multi-scale methods. International Journal for Numerical Methods in Engineering; 50:993-1013.

16. Feyel F (2003). A multi-level finite element method to describe the response of highly nonlinear structures using generalized continua. Computer Methods in Applied Mechanics and Engineering; 192: 3233-3244.

17. Geers MGD, Kouznetsova VG, Brekelmans WAM (2010). Multi-scale computational homogenization: Trends and challenges. Journal of Computational and Applied Mathematics; 234: 2175-2182.

18. Li S, Liu W-K (2002). Meshfree and particle methods and their applications. Applied Mechanics Review; 55: 1-34.

19. Zhang LT, Liu WK, Li SF, Qian D, Hao S (2002), Survey of multi-scale and meshfree particle methods. Meshfree Methods for Partial Differential Equations, eds, Griebel M, Schweitzer MA, Lecture Notes in Computational Science and Engineering: 441-458.

20. Babuska I, Banarjee U, Osborn JE (2003). Survey of meshless and generalized finite element methods: A unified approach. Acta Numerica; 12: 1-125.

21. Huerta A, Belytschko T, Fernandez-Mendez S, Rabczuk T (2004). Meshfree methods. Encyclopaedia of Computational Mechanics; 5:1-49.

22. Haidar K, Dube, JF, Gilles P-C (2003). Modelling crack propagation in concrete structures with a two scale approach. International Journal for Numerical and Analytical Methods in Geomechanics; 45:601-620.

23. Mosler J (2005). On the efficient implementation of an elasto-plastic damage model for large scale analyses of material failure: a multiscale approach. Computers \& Structures; 83:369-382. 
24. Liu WK, Uras RA, Chen Y (1997). Enrichment of the finite element method with the reproducing kernel particle method. Journal of Applied Mechanics, ASME; 64(4):861-870.

25. Wagner GJ, Liu WK (2003). Coupling of atomistic and continuum simulations using bridging scale decomposition. Journal of Computational Physics; 190:249274.

26. Qian D, Wagner GJ, Liu WK (2004). A Multi-scale projection method for the analysis of carbon nanotubes. Computer Methods in Applied Mechanics and Engineering; 193: 1603-1632.

27. Liu WK, Park HS, Qian D, Karpov EG, Kadowaki H, Wagner GJ (2006). Bridging scale methods for nanomechanics and materials. Computer Methods in Applied Mechanics and Engineering; 195:1407-1421.

28. Kadowaki H, Liu WK (2004). Bridging multi-scale method for localization problems. Computer Methods in Applied Mechanics and Engineering; 193:32673302.

29. Ju GT, Kyriakides S (1992). Bifurcation and localization instabilities in cylindirical shells under bending-II. Predictions. International Journal of Solids and Structures 29: 1143-1171.

30. Song H-W, Tassoulas JL (1993). Finite element analysis of propagating buckles. International Journal for Numerical Methods in Engineering 36:3529-3552.

31. Ozkan I, Mohareb M (2009). Testing and Analysis of Steel Pipes under Bending, Internal Pressure, and Tension. Journal of Structural Engineering, ASCE 135 (2): 187-197 
32. Weicker K, Salahifar R, Mohareb M (2010). Shell analysis of thin-walled pipes II. Finite element formulation. International Journal of Pressure Vessels and Piping; 87:414-423.

33. Nowzartash F, Mohareb M (2004). An elasto-plastic finite element for steel pipelines. International Journal of Pressure Vessel and Piping; 81:919-930.

34. Bathe, K.-J. \& Almeida, C. A. (1980). A simple and effective pipe elbow element - linear analysis. Journal of Applied Mechanics, ASME, Vol. 47, pp.93-100.

35. Bathe, K.-J. \& Almeida, C. A. (1982), "A simple and effective pipe elbow element - intreraction effects", J. Applied Mechanics, ASME, Vol. 49, pp.165171.

36. Millittelo, C. and Huespe, A. E. (1988), "A displacement-based pipe elbow element", Computers and Structures, Vol. 29, No.2, pp. 339-343.

37. Yan, A. M., Jospin, R. J. and Nguyen, D. H. (1999), "An enhanced pipe elbow element - Application in plastic limit analysis of pipe structures", International Journal For Numerical Methods in Engineering, Vol. 46, pp. 409-431.

38. Erkmen RE, (2012). Bridging multi-scale approach to consider the effects of local deformations in the analysis of thin-walled members. Accepted for publication in Computational Mechanics.

39. Cook RD (1990). Simulating curved elements by offsets: rationale and application to shells of revolution. Engineering Computations 7:79-80.

40. Ibrahimbegovic A, Taylor RL, Wilson EL (1990). A robust quadrilateral membrane finite element with drilling degrees of freedom. International Journal for Numerical Methods in Engineering; 30:445-457.

41. Hughes TJR, Brezzi F (1989). On drilling degrees of freedom. Computer Methods in Applied Mechanics and Engineering; 72:105-121. 
42. Cook RD, Malkus DS, Plesha ME, Witt RJ (2002). Concepts and applications of finite element analysis. John Wiley \& Sons, fourth edition.

43. Amabili, M (2015). Non-linearities in rotation and thickness deformation in a new third-order thickness deformation theory for static and dynamic analysis of isotropic and laminated doubly curved shells. International Journal of Non-linear Mechanics; 69:109-128.

44. Crisfield MA (1991). Non-linear finite element analysis of solids and structures, Vol. 1. Wiley, New York.

45. Marques JMMC (1984). Stress computation in elastoplasticity. Engineering Computations 1:42-51.

46. Batoz J-L, Tahar MB (1982). Evaluation of a new quadrilateral thin plate bending element. International Journal for Numerical Methods in Engineering; 18:16551677.

47. Fafard F, Beaulieu D and Dhatt G (1987). A rational model for distortional buckling of tapered members. Computers \& Structures; 25:183-190.

48. Argyris JH, Papadrakakis M, Karapitta L (2002). Elasto-plastic analysis of shells with the triangular element TRIC. Computer Methods in Applied Mechanics and Engineering; 191:3613-3636. 


\section{Appendix A Matrix of Shell Shape functions}

Matrix $\hat{\mathbf{X}}$ in Eq. (8) which relates the displacement fields to the nodal displacements is explicitly given in the following

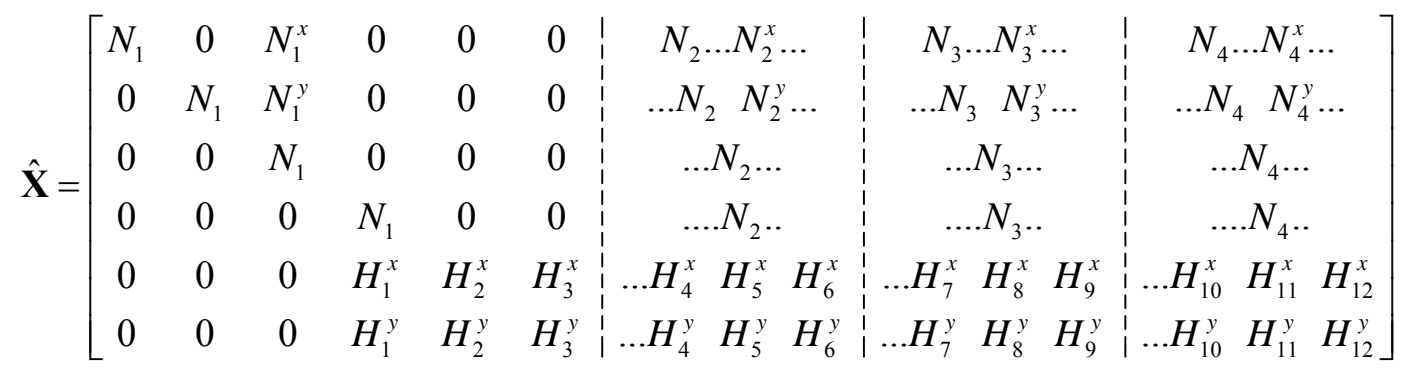

where the interpolation functions $N_{i}, H_{j}$ for membrane displacements and plate components are respectively provided in Sections A.1 and A.2

\section{A.1 Interpolation functions of the membrane component of the shell element}

In Eq. A. $1 N_{i}$ is the standard bilinear shape function defined as

$$
N_{i}=\frac{1}{4}\left(1+\xi_{i} \xi\right)\left(1+\eta_{i} \eta\right), i=1,2,3,4
$$

where $\xi=x / a$ and $\eta=y / b, a$ and $b$ are the half lengths of the rectangular member in $x$ and $y$ directions respectively. Coordinates $x$ and $y$ are measured from the middle of the rectangular element, i.e. $-1 \leq \xi \leq 1$ and $-1 \leq \eta \leq 1$. It should also be noted that $2 \times 2$ Gaussian quadrature was used for the numerical integration of both plate and membrane. Membrane related functions $N_{i}^{x}$ and $N_{i}^{y}$ according to Allman-type interpolation are defined as 


$$
\begin{aligned}
& N_{i}^{x}=\frac{1}{8}\left(y_{i j} N_{l}-y_{i k} N_{m}\right),(i=1,2,3,4) \\
& N_{i}^{y}=\frac{1}{8}\left(x_{i j} N_{l}-x_{i k} N_{m}\right),(i=1,2,3,4)
\end{aligned}
$$

in which

$$
\begin{aligned}
& N_{m}=\frac{1}{2}\left(1-\xi^{2}\right)\left(1+\eta_{m} \eta\right),(m=8,5,6,7) \\
& N_{l}=\frac{1}{2}\left(1+\xi_{l} \xi\right)\left(1-\eta^{2}\right),(l=5,6,7,8) .
\end{aligned}
$$

where $x_{i j}=x_{j}-x_{i}, y_{i j}=y_{j}-y_{i}, l_{i j}{ }^{2}=x_{i j}{ }^{2}+y_{i j}{ }^{2},(i j=41,12,23,34)$ and $(i k=12,23,34,41)$.

\section{A.2 Interpolation functions of the plate component of the shell element}

Shape functions of the Discrete Kirchhoff Quadrilateral can be explicitly written as

$$
\begin{aligned}
& H_{1}^{x}=1.5\left(a_{5} N_{5}-a_{8} N_{8}\right), H_{2}^{x}=-N_{1}+c_{5} N_{5}+c_{8} N_{8}, H_{3}^{x}=b_{5} N_{5}+b_{8} N_{8}, H_{4}^{x}=1.5\left(a_{6} N_{6}-a_{5} N_{5}\right) \\
& H_{5}^{x}=-N_{2}+c_{6} N_{6}+c_{5} N_{5}, H_{6}^{x}=b_{6} N_{6}+b_{5} N_{5}, H_{7}^{x}=1.5\left(a_{7} N_{7}-a_{6} N_{6}\right), H_{8}^{x}=-N_{3}+c_{7} N_{7}+c_{6} N_{6} \\
& H_{9}^{x}=b_{7} N_{7}+b_{6} N_{6}, H_{10}^{x}=1.5\left(a_{8} N_{8}-a_{7} N_{7}\right), H_{11}^{x}=-N_{4}+c_{8} N_{8}+c_{7} N_{7}, H_{12}^{x}=b_{8} N_{8}+b_{7} N_{7} \\
& H_{1}^{y}=1.5\left(d_{5} N_{5}-d_{8} N_{8}\right), H_{2}^{y}=b_{5} N_{5}+b_{8} N_{8}, H_{3}^{y}=-N_{1}+e_{5} N_{5}+e_{8} N_{8}, H_{4}^{y}=1.5\left(d_{6} N_{6}-d_{5} N_{5}\right) \\
& H_{5}^{y}=b_{6} N_{6}+b_{5} N_{5}, H_{6}^{y}=-N_{2}+e_{6} N_{6}+e_{5} N_{5}, H_{7}^{y}=1.5\left(d_{7} N_{7}-d_{6} N_{6}\right), H_{8}^{y}=b_{7} N_{7}+b_{6} N_{6} \\
& H_{9}^{y}=-N_{3}+e_{7} N_{7}+e_{6} N_{6}, H_{10}^{y}=1.5\left(d_{8} N_{8}-d_{7} N_{7}\right), H_{11}^{y}=b_{8} N_{8}+b_{7} N_{7}, H_{12}^{y}=-N_{4}+e_{8} N_{8}+e_{7} N_{7}
\end{aligned}
$$


in which $a_{k}=-\frac{x_{i j}}{l_{i j}{ }^{2}}, b_{k}=\frac{3 x_{i j} y_{i j}}{4 l_{i j}{ }^{2}}, c_{k}=\frac{\frac{1}{4} x_{i j}{ }^{2}-\frac{1}{2} y_{i j}{ }^{2}}{l_{i j}{ }^{2}}, d_{k}=-\frac{y_{i j}}{l_{i j}{ }^{2}}, e_{k}=\frac{-\frac{1}{2} x_{i j}{ }^{2}+\frac{1}{4} y_{i j}{ }^{2}}{l_{i j}{ }^{2}}$

$(k=5,6,7,8)(i=1,2,3,4),(j=2,3,4,1)$ and $(i j=12,23,34,41)$

\section{Appendix B}

In Eq. (15), the incremental strains $\delta \hat{\boldsymbol{\varepsilon}}$ were expressed in terms of the incremental nodal

displacements $\hat{\mathbf{d}}$ through $\delta \hat{\boldsymbol{\varepsilon}}=\hat{\mathbf{S}} \hat{\mathbf{B}} \delta \hat{\mathbf{d}}$ in which matrix $\hat{\mathbf{S}}$ can be written as

$$
\hat{\mathbf{S}}=\left[\begin{array}{l|l}
\mathbf{I} & -z \mathbf{I}
\end{array}\right]
$$

in which $\mathbf{I}$ is a $4 \times 4$ unit matrix and matrix $\hat{\mathbf{B}}$ can be written as

$$
\hat{\mathbf{B}}=\left[\begin{array}{c:c}
\hat{\mathbf{B}}_{m}+\widehat{\mathbf{B}}_{N m} & \widehat{\mathbf{B}}_{N b} \\
\hdashline \mathbf{0} & \hat{\mathbf{B}}_{b}
\end{array}\right]\left\{\begin{array}{c}
\mathfrak{I} \\
\hdashline \mathfrak{\aleph}
\end{array}\right\} \hat{\mathbf{X}}
$$

and

$$
\begin{aligned}
\widehat{\mathbf{B}}_{m}=\left[\begin{array}{ccccc}
1 & 0 & 0 & 0 & 0 \\
0 & 1 & 0 & 0 & 0 \\
0 & 0 & 1 & 1 & 0 \\
0 & 0 & -\frac{1}{2} & \frac{1}{2} & -1
\end{array}\right], \widehat{\mathbf{B}}_{b}=\left[\begin{array}{ccccccc}
0 & 0 & 0 & 0 & 1 & 0 & 0 \\
0 & 0 & 0 & 0 & 0 & 1 & 0 \\
0 & 0 & 0 & 0 & 0 & 0 & 1 \\
0 & 0 & 0 & 0 & 0 & 0 & 0
\end{array}\right] \widehat{\mathbf{B}}_{N m}=\left[\begin{array}{ccccc}
0 & 0 & 0 & \frac{\partial \hat{v}_{0}}{\partial x} & 0 \\
0 & 0 & 0 & 0 & 0 \\
0 & 0 & 0 & 0 & 0 \\
0 & 0 & 0 & 0 & 0
\end{array}\right] \\
\widehat{\mathbf{B}}_{N b}=\left[\begin{array}{ccccccc}
\frac{\partial \hat{w}_{0}}{\partial x} & 0 & 0 & 0 & 0 & 0 & 0 \\
0 & 0 & 0 & 0 & 0 & 0 & 0 \\
0 & 0 & 0 & 0 & 0 & 0 & 0 \\
0 & 0 & 0 & 0 & 0 & 0 & 0
\end{array}\right],
\end{aligned}
$$

and 


$$
\mathfrak{I}=\left[\begin{array}{cccccc}
\frac{\partial}{\partial x} & 0 & 0 & 0 & 0 & 0 \\
0 & \frac{\partial}{\partial y} & 0 & 0 & \frac{\partial f}{\partial r} & 0 \\
\frac{\partial}{\partial y} & 0 & 0 & 0 & 0 & 0 \\
0 & \frac{\partial}{\partial x} & 0 & 0 & 0 & 0 \\
0 & 0 & 0 & 0 & 0 & 1
\end{array}\right],
$$

$\aleph=\left[\begin{array}{cccccc}0 & 0 & 0 & \frac{\partial}{\partial x} & 0 & 0 \\ 0 & 0 & 0 & \frac{\partial}{\partial y} & 0 & 0 \\ 0 & 0 & 0 & 0 & 1 & 0 \\ 0 & 0 & 0 & 0 & 0 & 1 \\ 0 & 0 & 0 & 0 & \frac{\partial}{\partial x} & 0 \\ 0 & 0 & 0 & 0 & 0 & \frac{\partial}{\partial y} \\ 0 & 0 & 0 & \frac{\partial^{2}}{\partial x \partial y} & 0 & 0\end{array}\right]$

B. (7)-(8) 


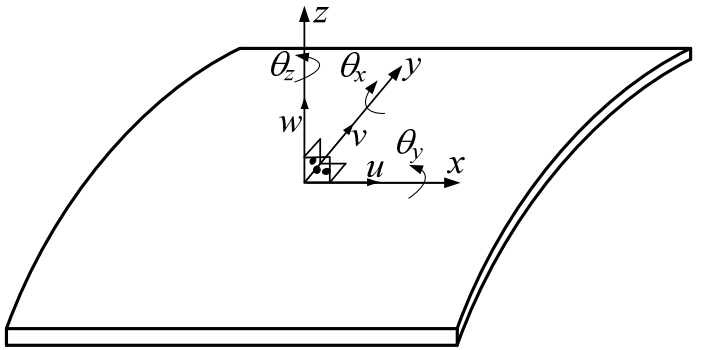

(a) Local deflections of the shell element formulation

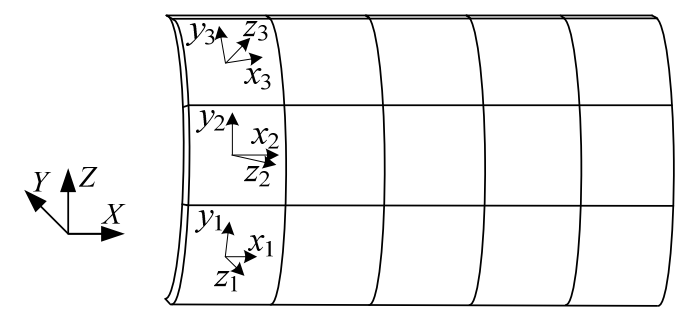

(c) Global vs. local coordinate system formulation

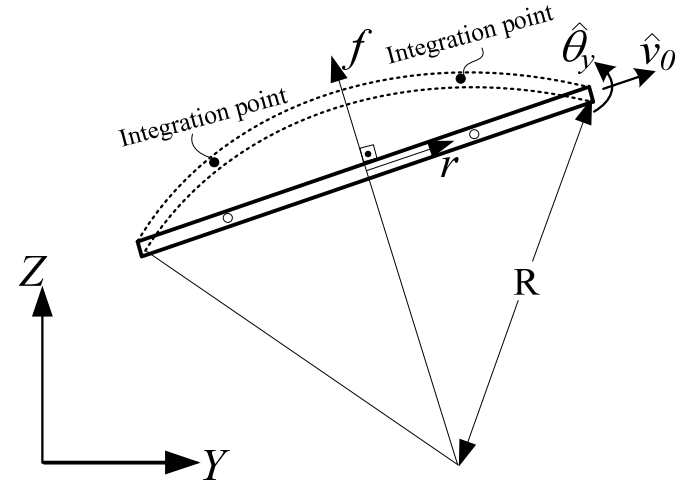

(b) Arch elevation used in shell

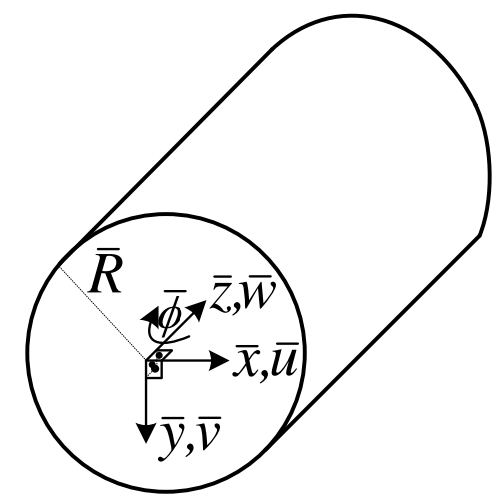

(d) Deflections of the beam-type

Fig.1: Deflections and coordinate systems

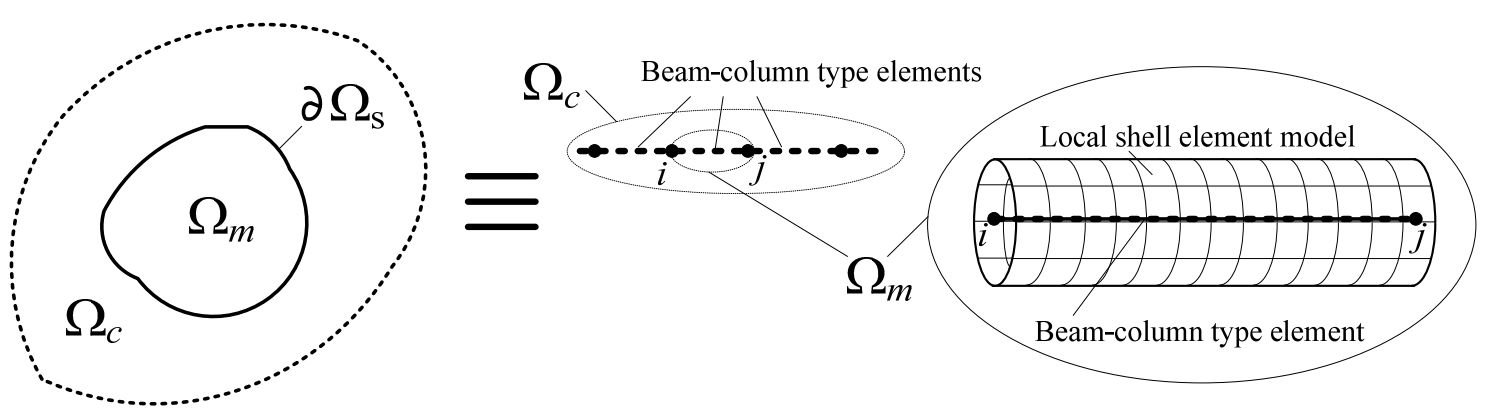

Fig.2: Decomposition of the analysis domain 


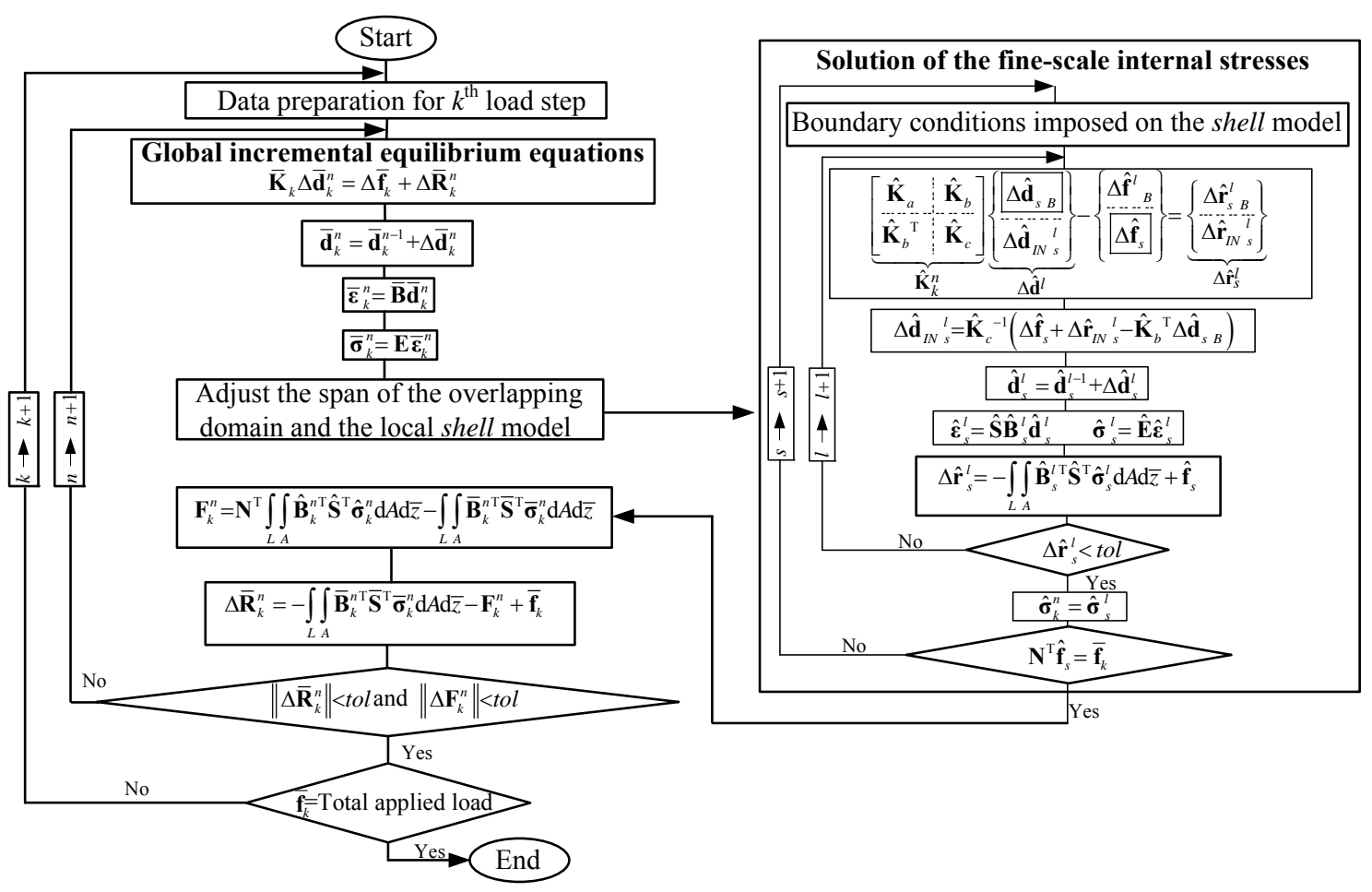

Fig.3: Flow-chart of the concurrent multi-scale analysis algorithm

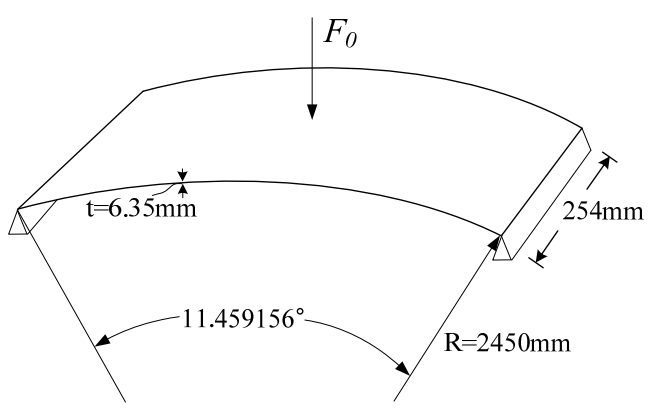

(a) Dimensions and loading middle

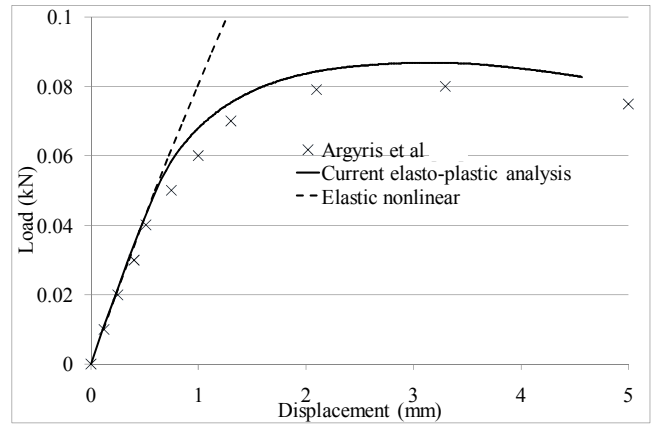

(b) Load-deflection relations in the

Fig.4: Description of the arch and load-deflection relations 


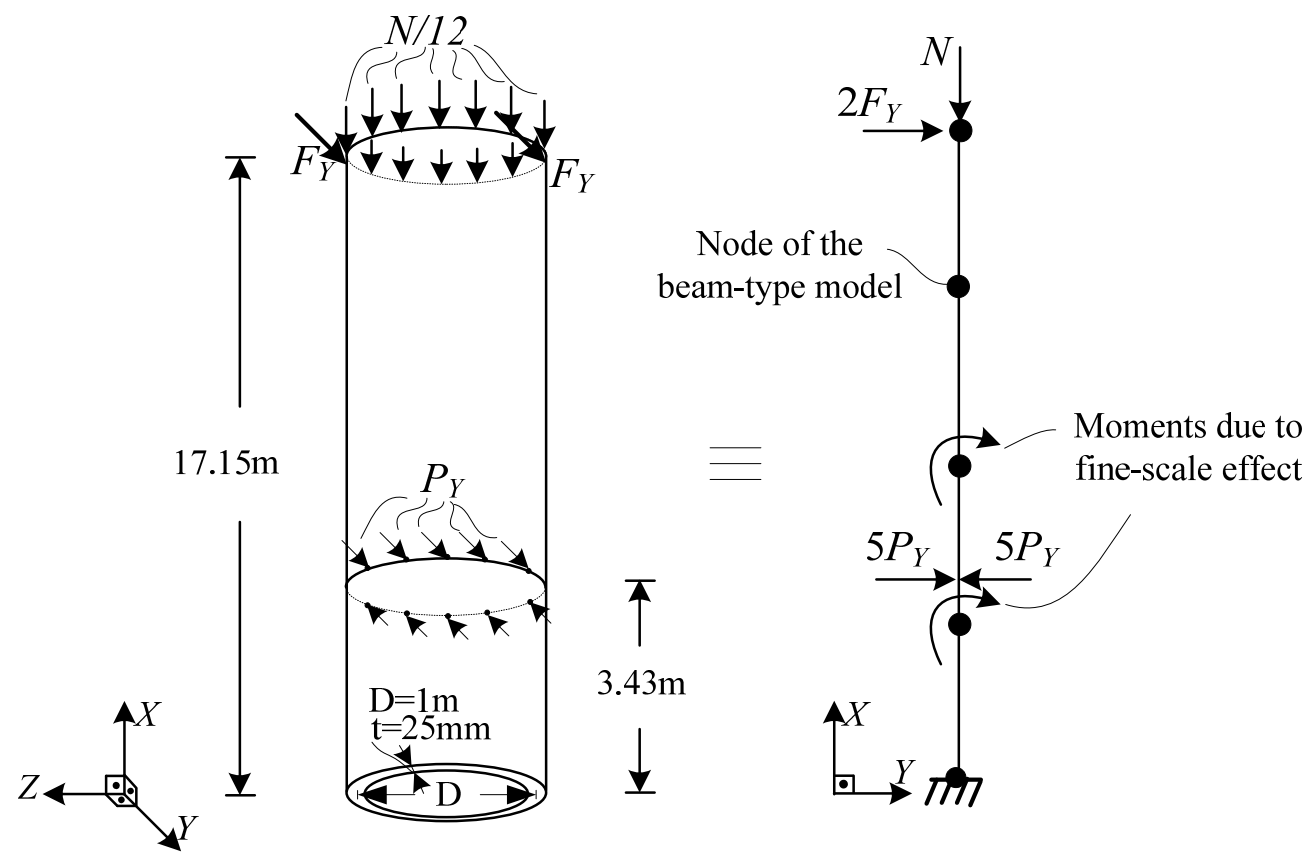

(a) Dimensions and loading of the pipe

(b) Equivalent multi-scale model

Fig.5: Description of the modelling of the pipe 


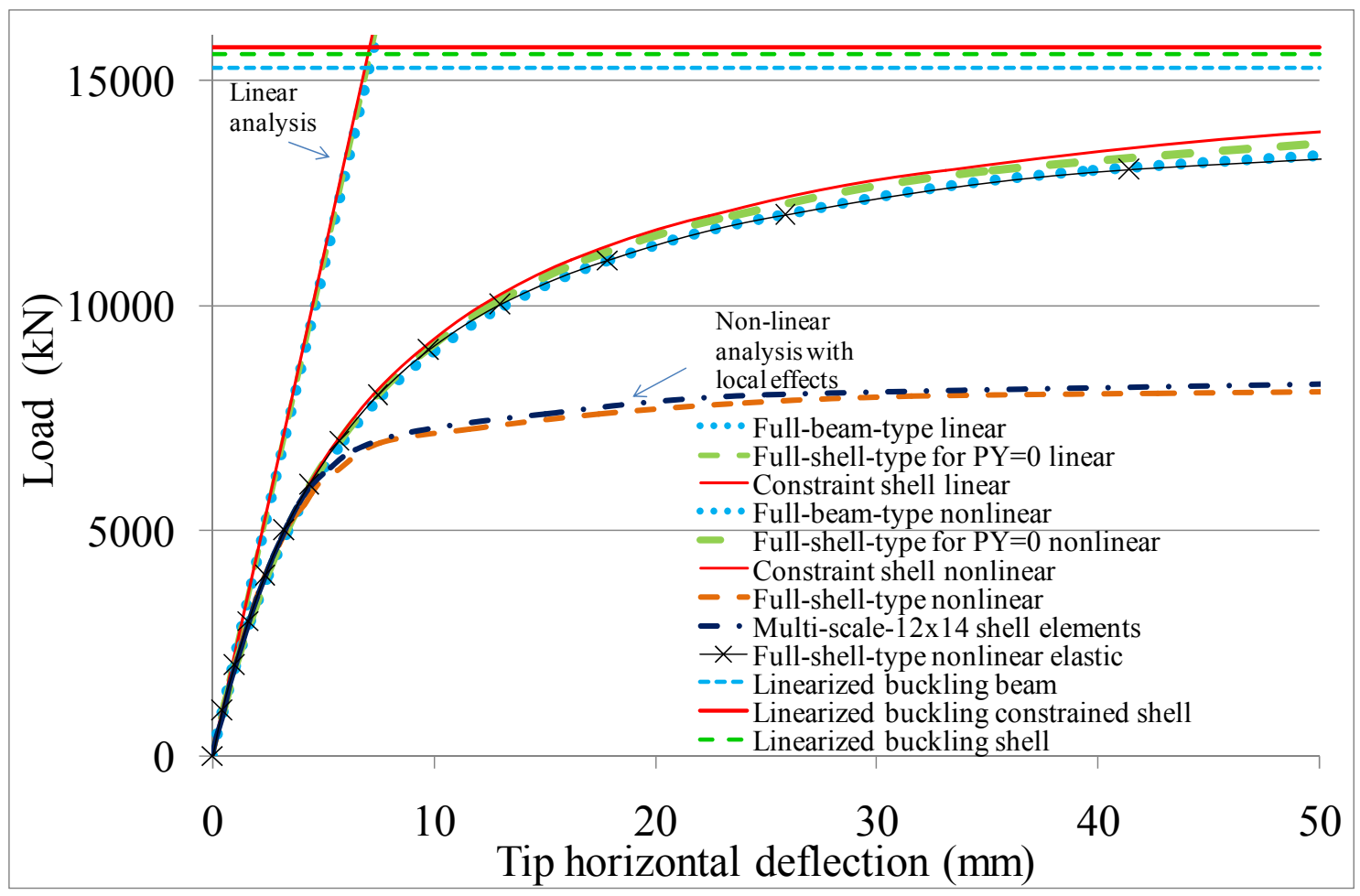

(a) Tip lateral deflection

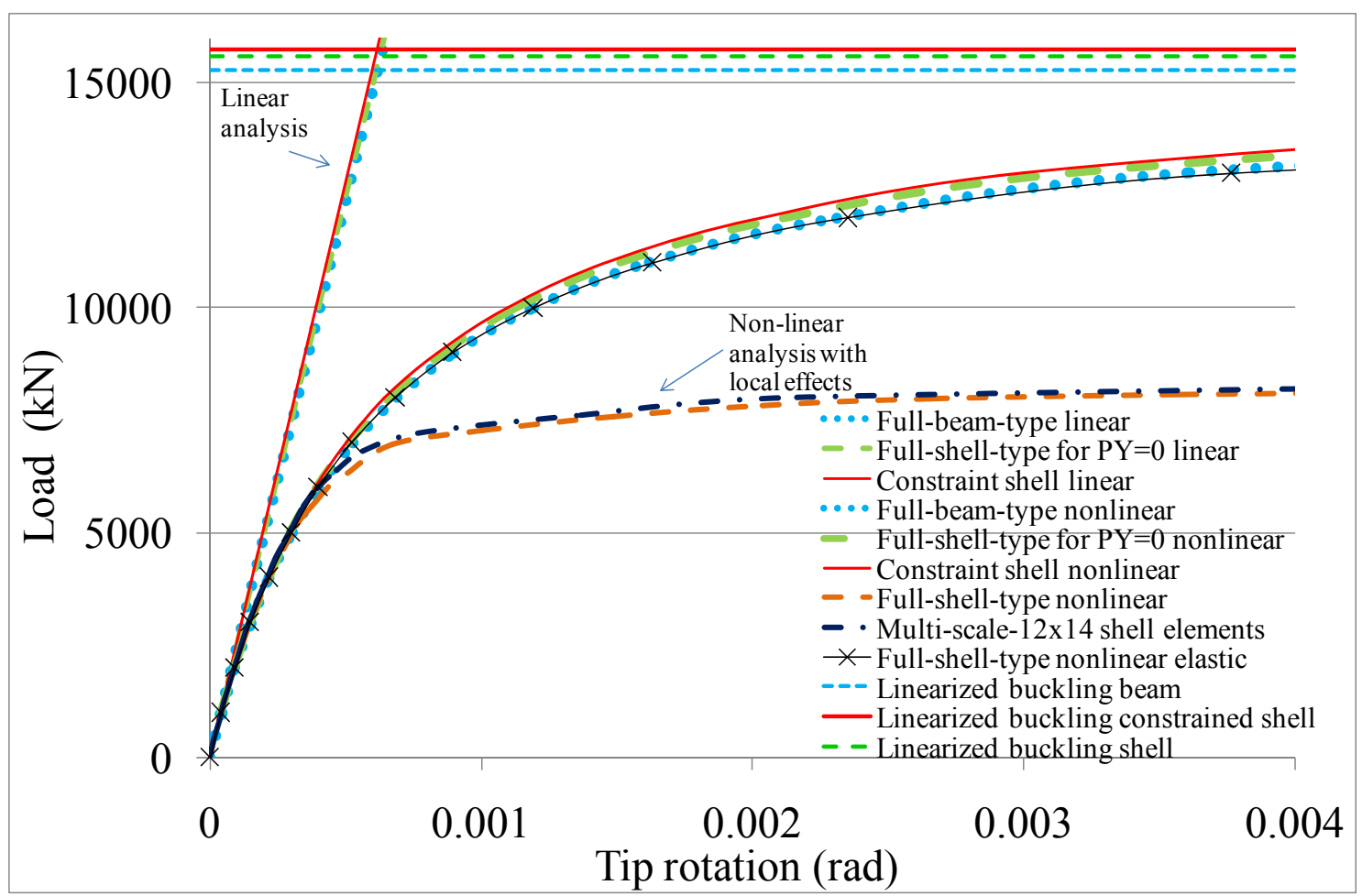

(b) Rotation at the tip

Fig.6: Load-deflection relations based on different modelling types 

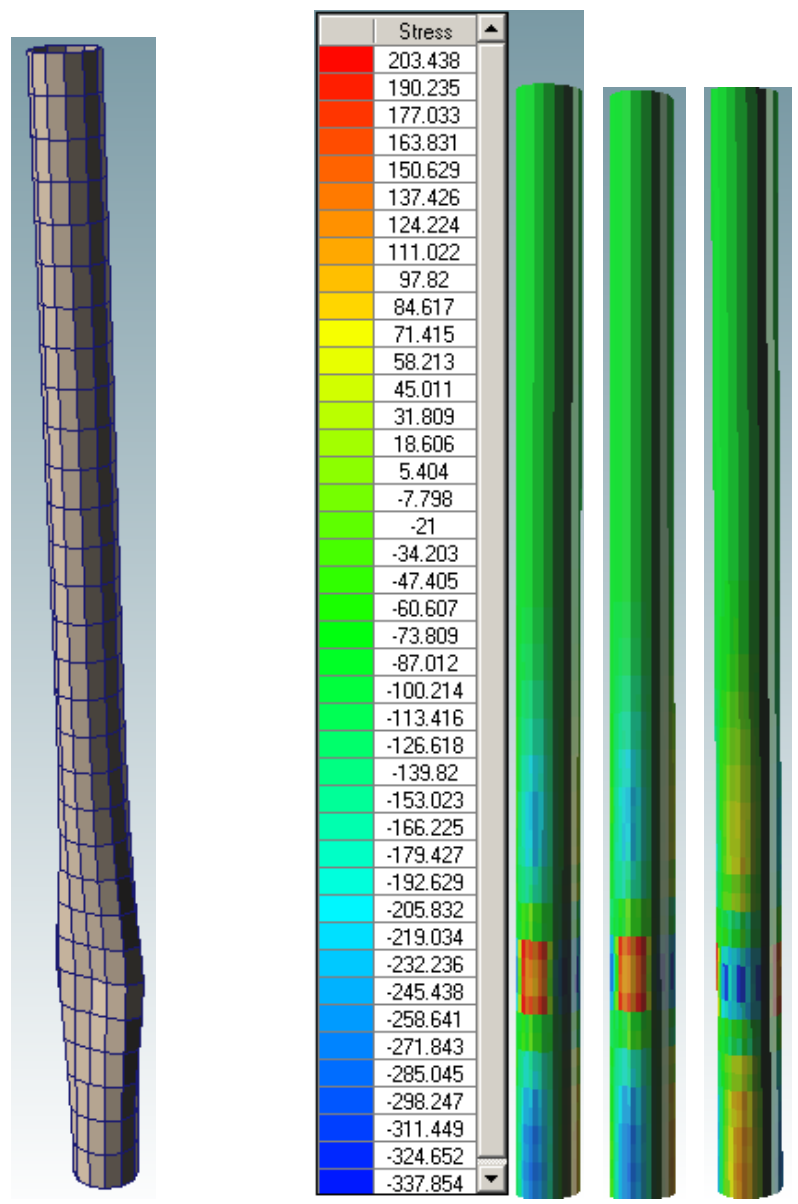

Front Back Side

Fig.7: Deformed shape and contour stress in longitudinal direction based on the shell-type nonlinear analysis (displacements scaled by a factor of 10 ) 


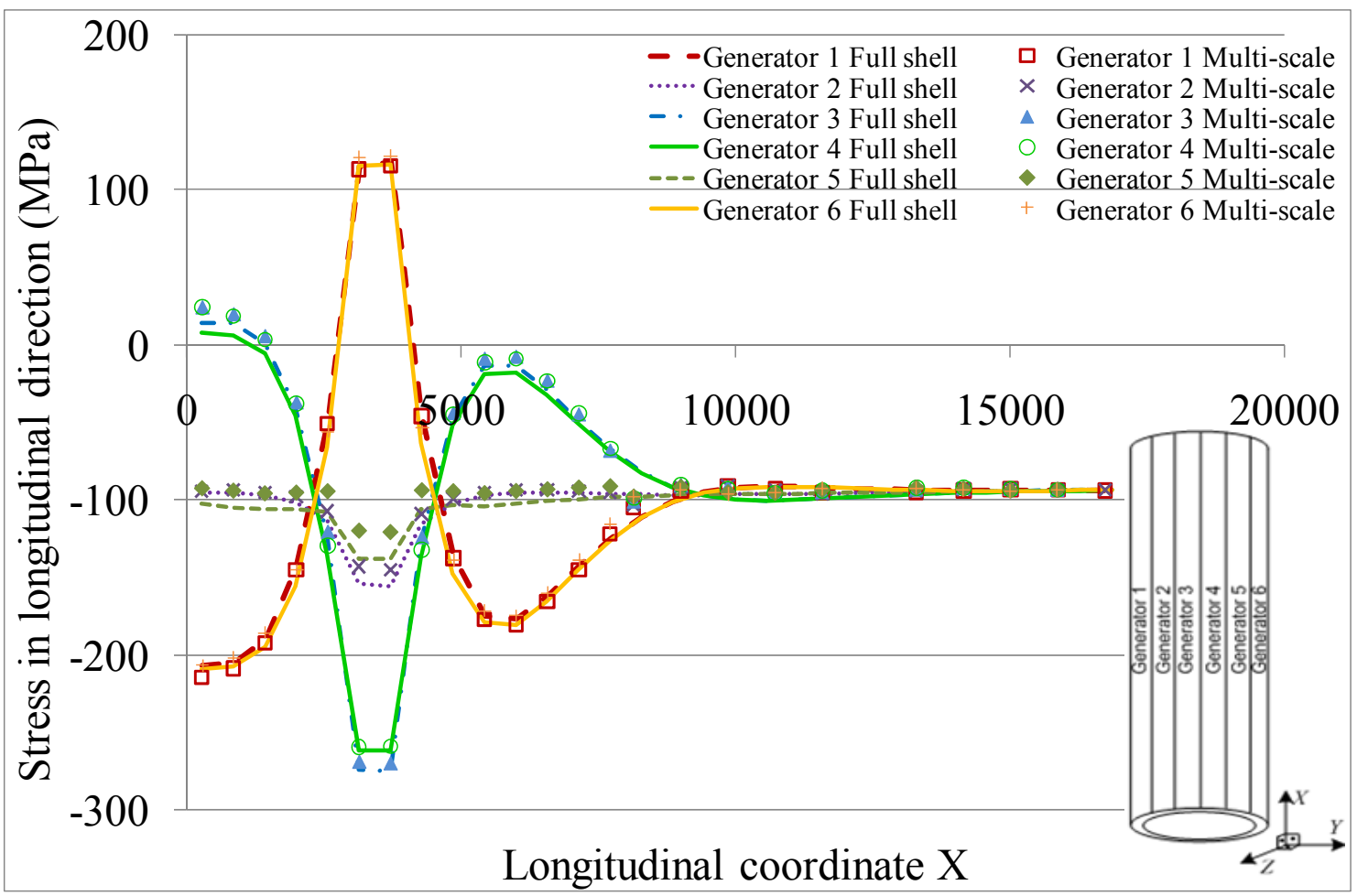

Fig. 8 Stress in longitudinal direction based on multi-scale and full shell-type analyses.

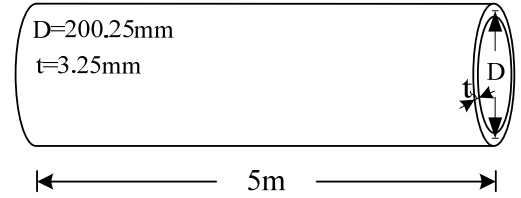

(a) Dimensions of the pipe

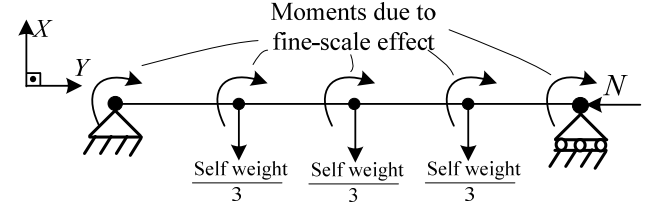

(b) Equivalent multi-scale model

Fig.9: Description of the modelling of the pipe 


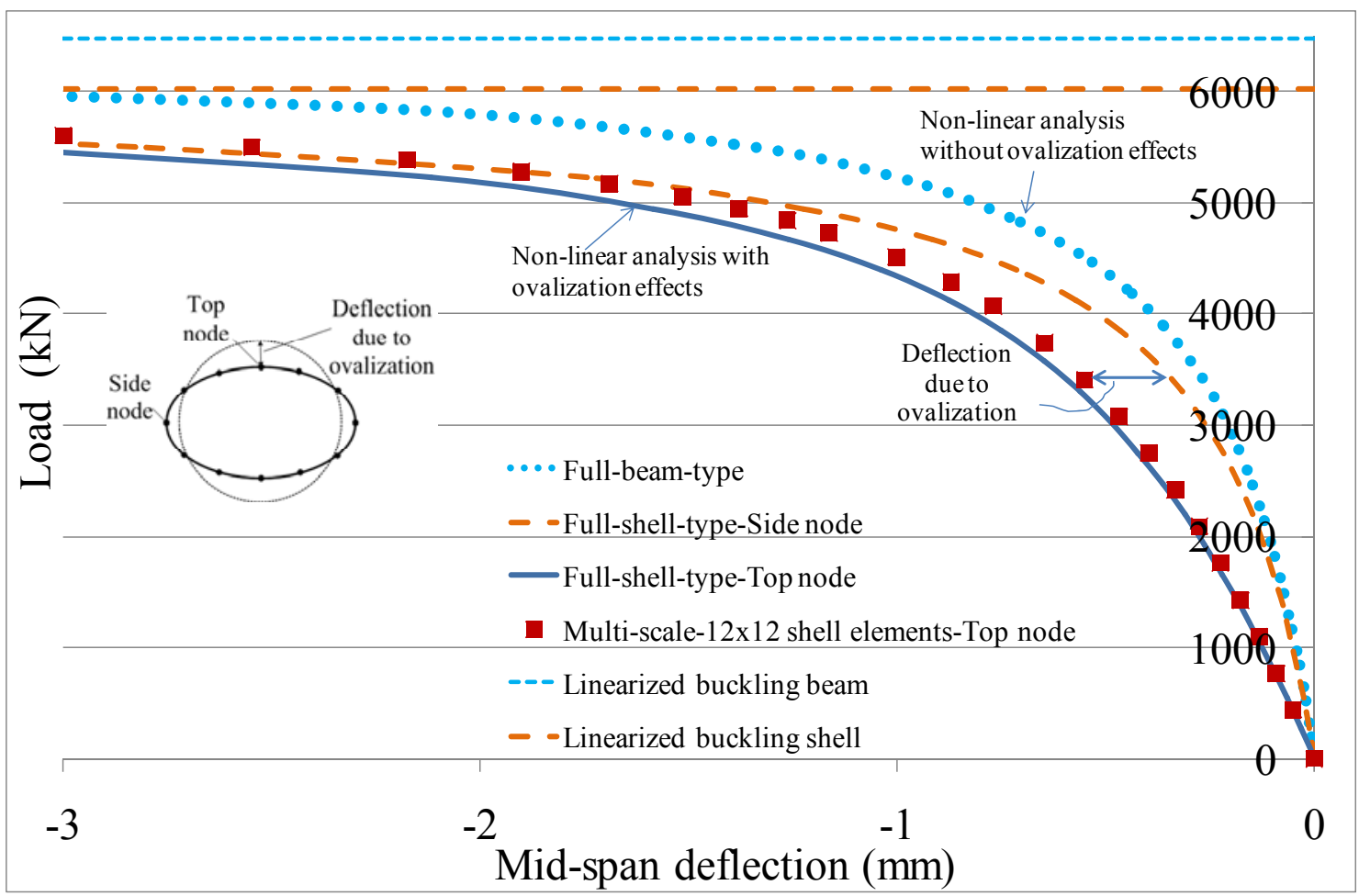

Fig.10: Load-deflection relations based on different modelling types 


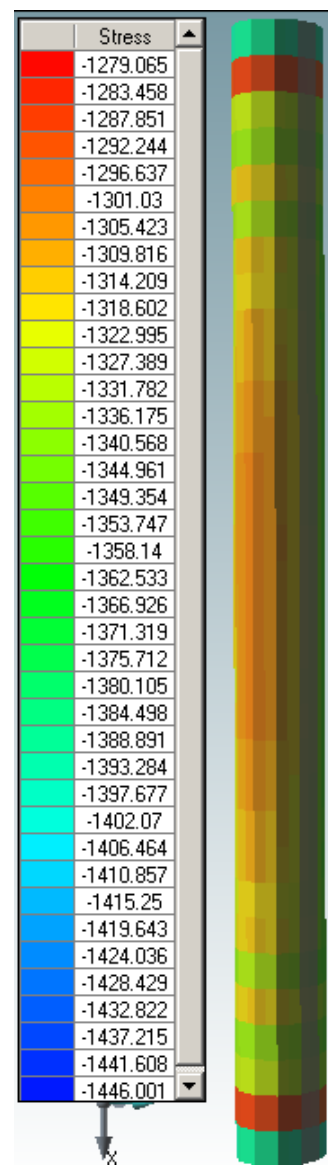

Front Back

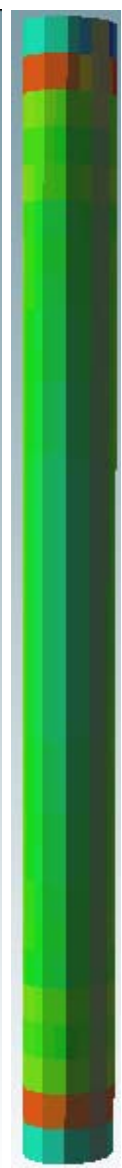

Fig.11: Deformed shape and stress contour based on shell-type nonlinear analysis (displacements scaled by a factor of 30 ) 


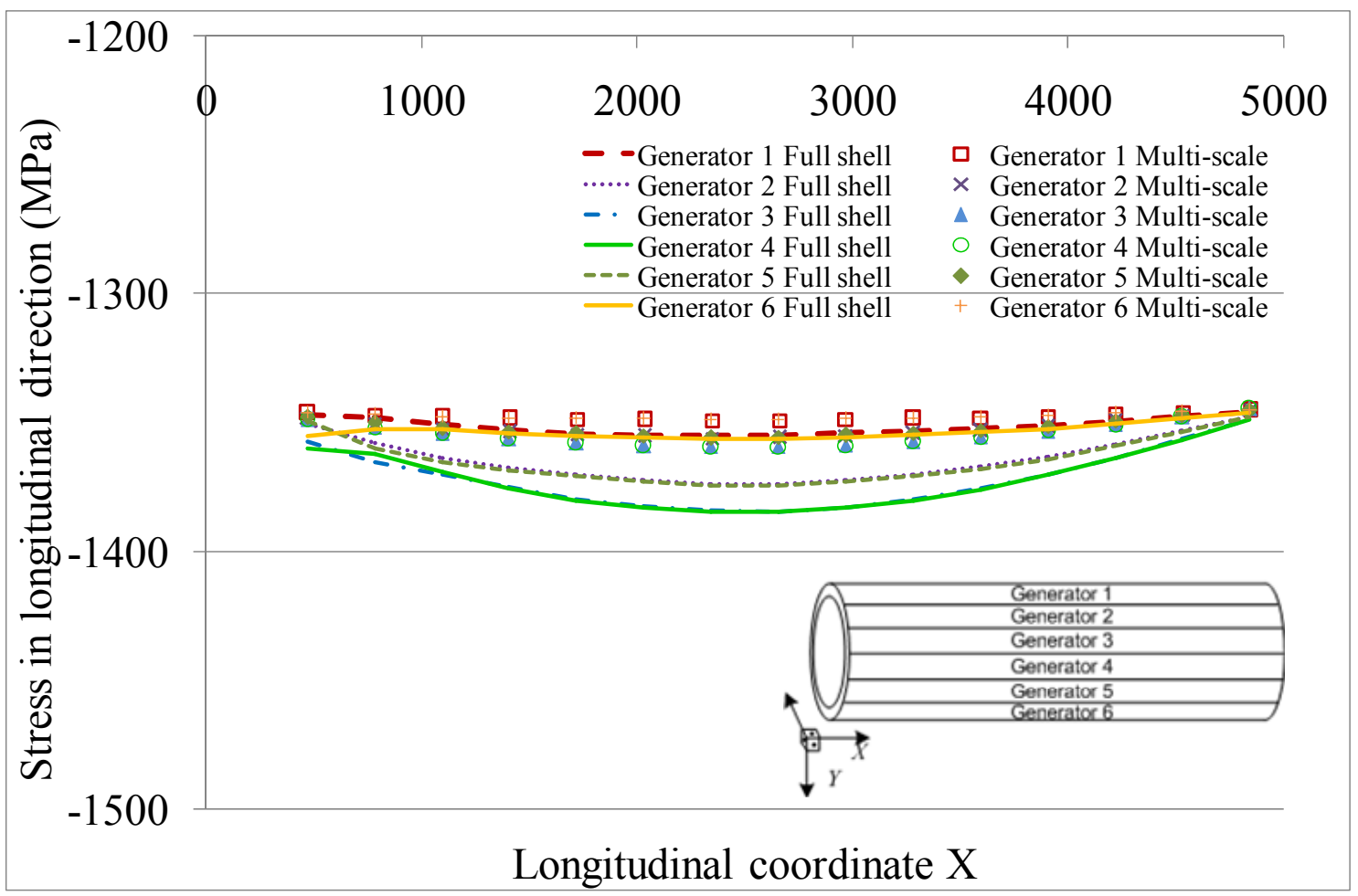

Fig.12 Stress in longitudinal direction based on multi-scale and full shell-type analyses. 